\title{
Conception d'un ascenseur à poissons adapté à l'alose (Alosa alosa) sur un grand cours d'eau: l'ascenseur de Golfech sur la Garonne
}

\author{
F. Travade ${ }^{(1)}$, M. Larinier ${ }^{(2)}$, D. Trivellato ${ }^{(3)}$ et J. Dartiguelongue ${ }^{(4)}$ \\ (1) EDF - Etudes et Recherches 6, quai Watier 78400 Chatou. \\ (2) CSP-CEMAGREF (GHAAPPE) BP 26, 31321 Castanet-Tolosan. \\ (3) Institut de Mécanique des Fluides (GHAAPPE), avenue Camille-Soula 31300 Toulouse. \\ (4) SCEA, 300, rue Henri-Desbals 31100 Toulouse.
}

Résumé. - Depuis 1971, année de mise en service de l'aménagement hydroélectrique de Golfech sur la Garonne, le passage de l'alose (alosa alosa) et des autres espèces migratrices à l'amont de l'ouvrage était très limité voire impossible.

Dans cet article, les auteurs rapportent les principaux critères utilisés pour le dimensionnement d'un ascenseur à poissons implanté au droit de l'usine de Golfech.

Sa conception est directement inspirée de celle de l'ascenseur à poissons du barrage de Holyoke sur la rivière Connecticut (USA). Deux modèles hydrauliques ont été utilisés pour choisir la situation des entrées et optimiser l'attractivité de l'ouvrage et les conditions hydrodynamiques dans la partie basse du dispositif.

L'ascenseur a été construit en 1986-1987.

Les poissons migrateurs sont attirés dans un grand bassin de stabulation par un débit d'attrait et piégés à l'aide d'une grille articulée jouant le rôle de nasse anti-retour. Cette grille, mécanisée et montée sur un chariot, sert également à pousser les poissons audessus d'une cuve située à l'extrémité amont du bassin de stabulation.

Le débit d'attrait est injecté au travers de grilles verticales situées à l'amont de la cuve et sur les parois latérales du bassin de stabulation. Les poissons, remontés dans la cuve, sont déversés dans un canal de transfert qui leur permet de rejoindre le canal d'amenée de l'usine.

L'ascenseur à poissons a été contrôlé en 1987 et 1988 à l'aide d'un dispositif de comptage vidèo automatisé de façon à en tester l'efficacité, à valider certains critères utilisés pour sa conception et à acquérir des informations sur les caractéristiques migratoires des différents espèces. Au cours des premières années de contrôle, 25 espèces ont emprunté le dispositif et de 14000 à 66000 aloses par an ont été comptabilisées.

Mots-clés. - passe à poissons, ascenseur, Garonne, Golfech, poissons migrateurs, alose, Alosa alosa. 


\begin{abstract}
Since 1970's, as Golfech hydroelectric facility went into operation on the Garonne river, the passage upstream of the plant of allis shad (alosa alosa) and other migratory species was restricted or completely blocked.

The authors outline in this paper the design parameters and the functional features used for designing a fish elevator at Golfech power plant. Its design is directly based on the Holyoke fish elevator built on the Connecticut river (USA). Two hydraulic model studies were performed to choose the entrance setting up, and to optimize the attraction conditions and the hydraulic patterns in the holding pool and dissipation chambers.

The fish elevator was built in 1986-1987. Migratory fish is attracted into a large holding pool. A mechanical crowder is used to force fish to enter above the hopper situated at the upstream part of this holding pool. The attraction water enters through sides diffusers and gratings. Crowder gates at the entrance remain in a $V$-trap arrangement that prevent movement back out through the entrance. Fish collected in the hopper pool are released in an exit channel with low downstream velocities. This fish elevator was monitored with an automatic video recording system to test its efficiency, to acquire data on migratory pattern of the different species, as well as to validate some design criteria of such a fish facility. During the first years of testing, 25 species of fish and from 14000 to 66000 shad were counted at the viewing window.
\end{abstract}

Keys words. - Fishpass, fish elevator, Garonne river, Golfech, migratory fish, shad, Alosa alosa

\section{1. - INTRODUCTION}

L'aménagement hydroélectrique de Golfech, construit en 1971, s'est avéré difficilement franchissable, voire infranchissable par les poissons migrateurs, malgré l'installation, au barrage, de deux passes à poissons de type "écluse Borland". Les raisons essentielles en sont, pour une part, la mauvaise conception des écluses, mais surtout l'attraction préférentielle des migrateurs au pied de l'usine (Belaud et Labat 1984, Belaud et al. 1985), dépourvue de passe, du fait de la disparité entre le débit turbiné $\left(540 \mathrm{~m}^{3} / \mathrm{s}\right)$ et le débit restitué par le barrage dans le bras court-circuité (de $10 \mathrm{~m}^{3} / \mathrm{s}$ à $20 \mathrm{~m}^{3} / \mathrm{s}$ ).
Diverses études ichtyologiques réalisées dans les années 80 ont mis en évidence les risques de disparition de la population d'alose du fait de la réduction importante des frayères (Cassou-Leins et Cassou-Leins 1981) et ont confirmé le blocage des migrateurs à l'aval de l'usine hydroélectrique (Belaud et Labat 1984). Dès 1981, des opérations de capture des migrateurs à l'aval de l'usine et de transfert manuel en amont ont été conduites de façon à examiner les possibilités de recolonisation des frayères amont. Elles ont permis de recueillir des informations sur l'abondance et le comportement des migrateurs à l'aval du site de Golfech (Belaud et al. 1985, Belaud et Labat 1991). 
En parallèle à ces actions, EDF a initié des études destinées à examiner la faisabilité d'un ouvrage de franchissement à l'usine de Golfech. La complexité du problème, notamment concernant le franchissement de l'alose qui s'avère généralement beaucoup plus problématique que celui des autres migrateurs, a conduit à créer un groupe de travail pluridisciplinaire comprenant des biologistes, des hydrauliciens et des spécialistes de Génie civil. Plusieurs organismes ont été associés à ce groupe de travail: le CEMAGREF, le CSP, I'ENSAT, I'IMFT, la SOGREAH et EDF(1).
Le présent article présente les raisons du choix de l'ouvrage de franchissement à Golfech, les critères de conception de ce type de passe, sa réalisation, et les premiers résultats de son fonctionnement.

\section{2. - LE SITE DE GOLFECH}

L'aménagement hydroélectrique de Golfech (fig 1), se compose d'un barrage mobile court-circuitant une quinzaine de kilomètres de Garonne (débit

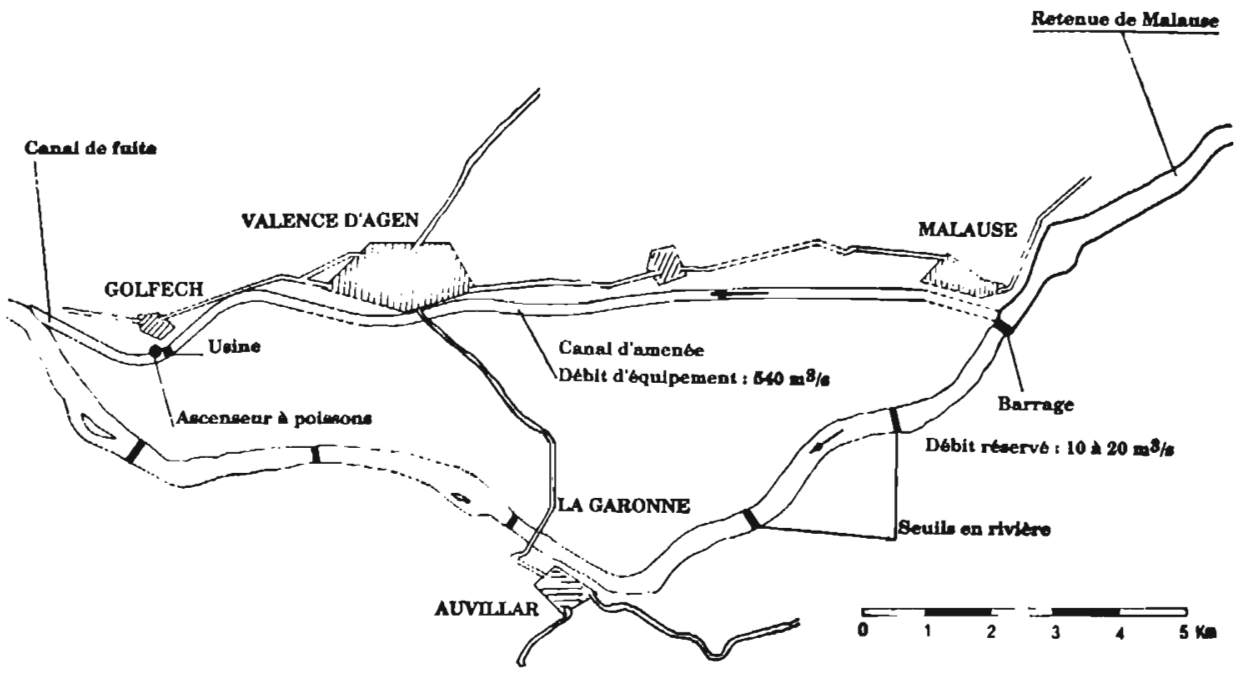

Fig. 1. - Plan d'ensemble de l'aménagement hydroélectrique de Golfech.

Fig. 1. - Golfech hydroelectric facility

(1) CEMAGREF : Centre du Machinisme Agricole, du Génie Rural et des Eaux et Forêts.

CSP : Conseil Supérieur de la Pêche.

ENSAT : Ecole Nationale Supérieure Agronomique de Toulouse.

IMFT : Institut de Mécanique des Fluides de Toulouse.

SOGREAH : Société Grenobloise d'Aménagements Hydrauliques.

EDF : Electricité de France. 
réservé de $7 \mathrm{~m}^{3} / \mathrm{s}$ jusqu'en 1981 et entre $10 \mathrm{~m}^{3} / \mathrm{s}$ et $20 \mathrm{~m}^{3} / \mathrm{s}$ suivant la période de l'année par la suite) pour alimenter, par un canal d'amenée de $10 \mathrm{Km}$ de longueur, l'usine équipée de trois groupes bulbes turbinant un débit maximal de $540 \mathrm{~m}^{3} / \mathrm{s}$. Le débit turbiné est restitué en Garonne par un canal de fuite de $2 \mathrm{Km}$ de longueur.

La chute à l'usine est de $17 \mathrm{~m}$ en étiage (cote amont 64,50 NGF, cote aval $47,50 \mathrm{NGF}$ ). La variation du niveau amont est d'environ 1 mètre en exploitation normale (cote 63,50 à 64,50 NGF) celle du niveau aval est de 2,60 m entre le niveau d'étiage et le niveau correspondant à un débit en Garonne de $1000 \mathrm{~m}^{3} / \mathrm{s} \quad(50,10 \mathrm{NGF})$ retenu comme limite de fonctionnement de l'ouvrage de franchissement des migrateurs.

Au droit des aspirateurs des turbines (fig. 2), le canal de fuite présente sur $25 \mathrm{~m}$ de longueur, une section rectangulaire de $38 \mathrm{~m}$ de largeur (haut des bajoyers à la cote $54,50 \mathrm{NGF}$ ) puis s'évase en une section trapézoïdale de $65 \mathrm{~m}$ de largeur en étiage à $127 \mathrm{~m}$ de largeur en crue.

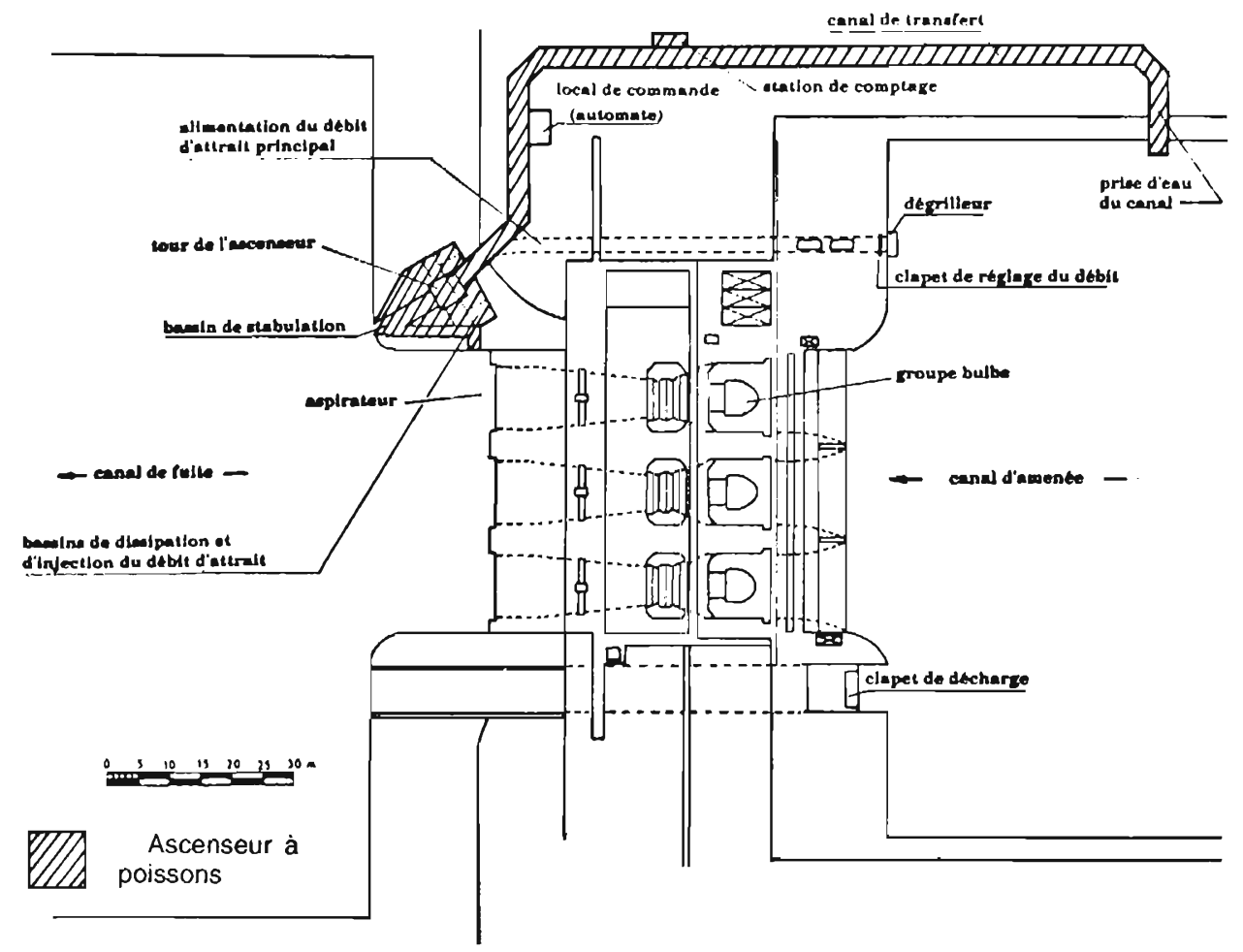

Fig. 2. - Usine hydrólectrique de Golfech. Situation de l'ascenseur à poissons.

Fig. 2. - Golfech powerhouse. Location of the fish elevator 


\section{3. - CHOIX DU TYPE DE DISPOSITIF \\ DE FRANCHISSEMENT}

Le dispositif de franchissement devait être utilisable par l'ensemble des poissons migrateurs présents en $\mathrm{Ga}$ ronne (alose, saumon, truite de mer, lamproie, anguille). Une attention toute particulière a été portée à l'alose (Alosa alosa), migrateur non seulement le plus abondant sur le site, mais également - si l'on se réfère à l'expérience acquise sur l'alose américaine - le plus exigeant vis à vis du type de passe utilisé, et des critères de conception et de fonctionnement.

Deux solutions étaient, à priori, envisageables, la passe à bassins successifs et l'ascenseur à poissons. L'expérience française sur les dispositifs de franchissement spécifiques à l'alose était, au départ du projet, pratiquement inexistante. D'après les quelques observations effectuées sur le Rhône (Larinier, 1981) et la Garonne (Cassou-Leins et Cassou-Leins 1981, Douchement 1981), la biologie et surtout le comportement migratoire de l'alose française (Alosa alosa) semblaient très voisins de ceux de l'alose américaine (Alosa sapidissima). Il a donc paru raisonnable de s'inspirer de l'expérience américaine pour concevoir un dispositif de franchissement sur le site de Golfech.

Le dispositif de franchissement qui se révèle le plus efficace pour l'alose américaine sur la Côte Est des Etats Unis est l'ascenseur à poissons: de 300000 à 400000 aloses par an transitent à l'ascenseur d'Holyoke sur la rivière Connecticut, fleuve d'une importance comparable à la Garonne (Larinier et Travade 1982). Par contre, la conception des passes à bassins pour l'alose s'est avérée beaucoup plus délicate pour cette même espèce : l'alose est en effet très sensible à l'hydrodynamique des bassins (répulsion devant les écoulements trop turbulents, les jets plongeants et les orifices noyés, blocage dans les zones d'eau mortes et les encoignures).

Ces divers problèmes ont été mis en évidence sur des passes à bassins construites dans les annèes 1980 , notamment celles équipant des aménagements situés sur la rivière Connecticut (usines hydroélectriques de Turners Falls et de Vernon) (Larinier et Travade 1982). L'efficacité de ces passes s'est révélée très décevante les premières années et plusieurs modifications conséquentes ont été rendues nécessaires (Rideout et al., 1985).

Pour ces diverses raisons, le choix s'est porté vers la solution de l'ascenseur dont l'intégration dans le site semblait par ailleurs poser a priori moins de problèmes que celle d'une passe classique.

\section{PRINCIPE \\ DE FONCTIONNEMENT \\ DE L'ASCENSEUR. CRITĖRES \\ DE DIMENSIONNEMENT}

Dans son principe, un ascenseur à poissons est un système mécanique qui consiste à capturer les poissons 
au pied de l'obstacle dans une cuve contenant une quantité d'eau appropriée à leur nombre, puis à remonter cette cuve et à la déverser en amont.

Les migrateurs sont attirés dans un bassin de stabulation (ou bassin de piégeage) par un "débit d'attrait" constitué d'une veine d'eau aux caractéristiques adaptées au comportement migratoire naturel et aux capacités de nage (vitesse et endurance) des espèces concernées. Ils y sont piégés par un système anti-retour puis, à intervalles réguliers, concentrés par une grille verticale mobile (mouvement de translation), au dessus de la cuve située à l'extrémité amont du bassin de stabulation. Une deuxième grille verticale mécanisée située à l'aval immédiat de la cuve, est alors descendue pour empêcher les poissons d'accéder sous cette cuve lorsque celle-ci est en manœuvre.

La cuve est remontée par un treuil puis vidangée dans le bief amont grâce à un dispositif approprié. Lorsque l'ascenseur ne peut être situé à l'aplomb de la retenue, le déversement s'effectue dans un canal relié au plan d'eau. On incite le poisson à en sortir en assurant dans le canal un courant d'une vitesse suffisante.

Plusieurs types de critères sont à prendre en compte pour la conception et le dimensionnement de tels ouvrages. Ils résultent essentiellement de l'expérience nord-américaine (Rizzo 1968, 1969; Bell, 1973; Dalley, 1980; Travade 1982):
- attraction des migrateurs: le débit délivré dans l'ouvrage pour y attirer les migrateurs doit être, comme pour tous les types de dispositifs de franchissement, fonction du débit du cours d'eau et de la configuration du site. En règle générale, il est de l'ordre de $1 \%$ à $5 \%$ du débit fluvial (Larinier 1983). L'écoulement à l'entrée de l'ouvrage doit avoir une certaine vitesse destinée à inciter les poissons à y pénétrer. On maintient à cet effet, à l'entrée, une chute de $0,2 \mathrm{~m}$ à $0,4 \mathrm{~m}$ (ce qui correspond respectivement à des vitesses voisine de $2 \mathrm{~m} / \mathrm{s}$ à $2,8 \mathrm{~m} / \mathrm{s}$ ) suivant les espèces considérées.

- capture et stabulation: le bassin de capture et de stabulation des poissons doit respecter des critères de vitesse et de volume. La vitesse maximale, fonction des espèces est de $0,3 \mathrm{~m} / \mathrm{s}$ à $0,6 \mathrm{~m} / \mathrm{s}$. Le volume $\mathrm{mi}$ nimal du bassin est fonction du nombre maximal instantané de poissons susceptibles d'être présents dans l'ouvrage : 15 litres par alose. Plus récemment, ce critère a été porté à 30 litres par alose (Rizzo 1986). Pour éviter l'affolement des poissons piégés et les blessures qui peuvent en résulter, ce bassin doit par ailleurs avoir des dimensions minimales: pour l'alose, profondeur minimale de $1,2 \mathrm{~m}$, largeur minimale de $2 \mathrm{~m}$ à $2,5 \mathrm{~m}$.

- cuve: de même que pour le bassin de stabulation, le volume de la cuve est fonction du nombre maximal de poissons présents lors d'une remontée. Le critère adopté aux USA à l'époque de la conception de l'as- 
censeur de Golfech était de $3,2 \mathrm{l} / \mathrm{kg}$ de poisson (Dalley 1980), soit environ 5,7 litres par alose. Plus récemment, ce critère a été porté à $6 \mathrm{l} / \mathrm{kg}$ de poisson (Rizzo 1986) soit environ 10 litres par alose.

- canal de transfert: il doit être suffisamment large et profond pour ne pas occasionner de perturbations dans le comportement des poissons : profondeur minimale de $1 \mathrm{~m}$, largeur minimale de 1,5 m. La vitesse de l'écoulement doit inciter le poisson à remonter tout en restant en deçà des capacités de nage de l'ensemble des espèces susceptibles d'emprunter l'ouvrage. Cette vitesse doit être comprise entre $0,3 \mathrm{~m} / \mathrm{s}$ et $0,6 \mathrm{~m} / \mathrm{s}$.

Le principe de dimensionnement des volumes du bassin de stabulation et de la cuve, adopté aux USA par le Fish and Wildlife Service (Rizzo 1986) consiste à prendre en compte le nombre maximal de poissons pouvant se présenter dans l'ouvrage durant le cycle le plus bref que peut assurer l'installation et à appliquer à ce nombre les critères spécifiques de volume cités précédemment. Sur les cours d'eau où l'alose est présente, c'est généralement cette espèce qui détermine le dimensionnement de l'ouvrage: la migration de l'alose s'effectue en effet "en bancs" avec un pic journalier très prononcé conduisant à une abondance instantanée en général très supérieure à celle des autres espèces. Une correction de volume est appliquée pour tenir compte des autres espèces présentes en même temps que l'alose. Le volume nécessaire pour le bassin de stabulation et la cuve est donné par:

$$
\mathrm{V}=\mathrm{Nc} \times \mathrm{Vmin}+\mathrm{C} \text { avec: }
$$

Nc: nombre maximal de poissons durant un cycle

Vmin : volume requis par chaque alose

C : coefficient de correction tenant compte de la présence des autres espèces

Aux USA, où la migration de l'alose (Alosa sapidissima) est bien connue, une estimation du nombre maximal de poissons arrivant au cours du cycle est obtenue en considérant que le pic journalier correspond à $10 \%$ de la migration annuelle et que le pic horaire au cours de cette journée est de 15\% de l'abondance journalière. Lorsque l'on connaît la taille approximative de la population migrante, le nombre maximal de poissons arrivant au cours d'un cycle est donc donné par la formule :

$\mathrm{Nc}=(\mathrm{Nt} \times 0,1 \times 0,15 \times d) / 60$ avec :

$\mathrm{Nt}$ : nombre de poissons migrant annuellement

d: durée du cycle le plus bref (en minutes)

Les informations partielles obtenues lors des opérations de transfert manuel des aloses de 1980 à 1986 (Belaud et Labat 1991) ont mis en évidence une similitude du mode de migration de l'alose à Golfech (captures journalières de $4 \%$ à $17 \%$ des captures annuelles) et de celui de l'alose américaine. De ce fait, les critères utilisés aux USA ont été repris pour le dimensionnement de l'ascenseur de Golfech. 


\section{5. - ADAPTATION AU SITE DE GOLFECH}

\subsection{Implantation de l'ascenseur}

Les principales difficultés ont résidé dans l'intégration de l'ascenseur dans les ouvrages existants, dans la détermination des débits nécessaires à une attractivité suffisante du dispositif et dans la localisation des entrées.

La solution optimale, utilisée sur la plupart des grands ouvrages en Amérique du Nord, aurait consisté à disposer plusieurs entrées de part et d'autre de l'usine et au dessus des turbines. Elle a été écartée, malgré son intérêt, compte tenu de la difficulté d'installation d'une galerie collectrice au-dessus des aspirateurs : la cote de la dalle recouvrant les aspirateurs est en effet située une cinquantaine de centimètres au-dessus du niveau aval d'étiage. Cette limitation imposait donc de situer la ou les entrées d'un seul côté de l'usine. La difficulté à installer ces entrées en rive gauche, et les observations biologiques préalables (Belaud et Labat 1991) ont conduit à choisir une implantation en rive droite.

Une première étude sur modèle réduit physique a été effectuée à l'Institut de Mécanique des Fluides de Toulouse; elle a permis d'envisager plusieurs variantes concernant la situation de la ou des entrées de l'ascenseur au pied des turbines (IMFT, 1985; Larinier et Trivellato 1987; Belaud et al 1987).
L'entrée principale de l'ascenseur a été implantée à une vingtaine de mètres en aval du rejet des turbines, sur la paroi aval de la plate-forme, au niveau de l'élargissement (fig. 3). Dans cette configuration, l'écoulement provenant de l'ascenseur constitue un jet relativement bien protégé des turbulences (photo 1) de l'usine et débouche dans une zone où avait été observé le passage des aloses. Une entrée secondaire située latéralement, à l'aval immédiat des turbines, dans le sillage de la dalle recouvrant les aspirateurs, a également été prévue (photo 2) : elle permettait de ménager un tube de courant bien individualisé dans la zone la plus amont de blocage du poisson.

Une troisième entrée (photo 3 ), ménagée dans la partie centrale du bajoyer rive droite, a été abandonnée car le jet issu de cette entrée était rapidement cisaillé par l'écoulement des turbines venant recoller à la paroi.

Une étude fine des jets issus des entrées a mis en évidence que des débits voisins de $5 \mathrm{~m}^{3} / \mathrm{s}$ par entrée étaient indispensables à la création d'un attrait de portée suffisante.

Une seconde étude sur modèle réduit effectuée au Laboratoire SOGREAH a permis d'optimiser les écoulements à l'intérieur de la partie basse du dispositif (fig. 4), en particulier la dissipation du débit d'attrait et son injection dans le bassin de stabulation (Puyo et Venel 1987). Un ensemble de quatre vannes permettant d'ajuster les alimentations en eau entre l'amont et les parois latérales du bassin de stabulation a été ajouté par 


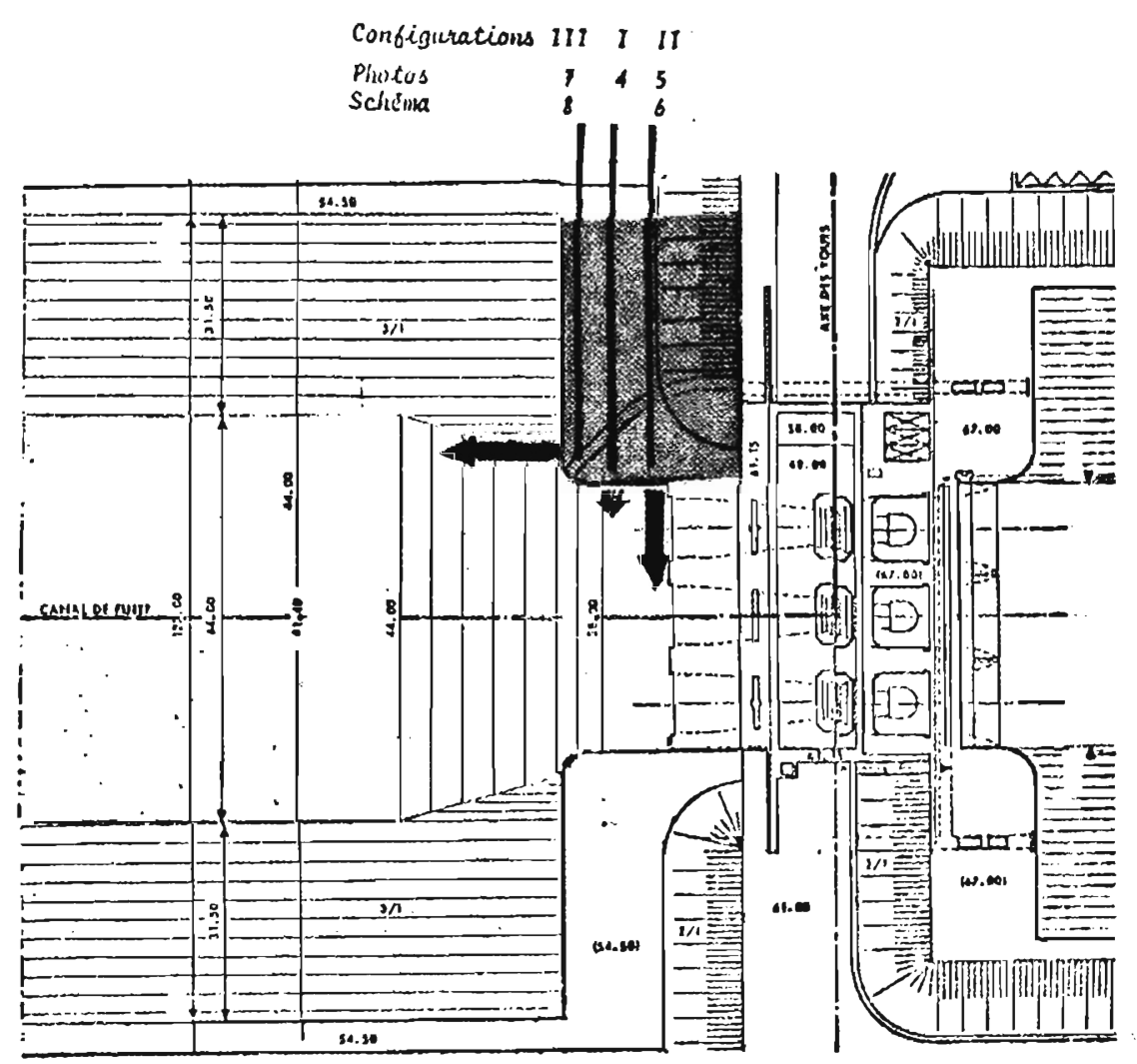

Fig. 3. - Implantation des entrées de l'ascenseur à poissons testées sur modèle réduit. Fig. 3. - Location of fish elevator entrances tested on hydraulic model

la suite de façon à permettre plus de souplesse dans les réglages des débits et à pouvoir moduler les conditions hydrodynamiques dans le bassin de stabulation et au droit de la nasse de capture.

\subsection{Dimensionnement}

Les volumes de la cuve et du bassin de stabulation ont été déterminés à l'aide des critères présentés au paragraphe 4 en prenant en compte une population maximale de 400000 aloses/an (Belaud et Labat 1991). En considérant, suite aux expérimentations réalisées auparavant sur le site, que les rythmes de migration de la grande alose sont identiques à ceux de l'alose américaine (pic journalier correspondant à environ $10 \%$ de la migration annuelle et pic horaire à $15 \%$ de la migration journalière) et en 


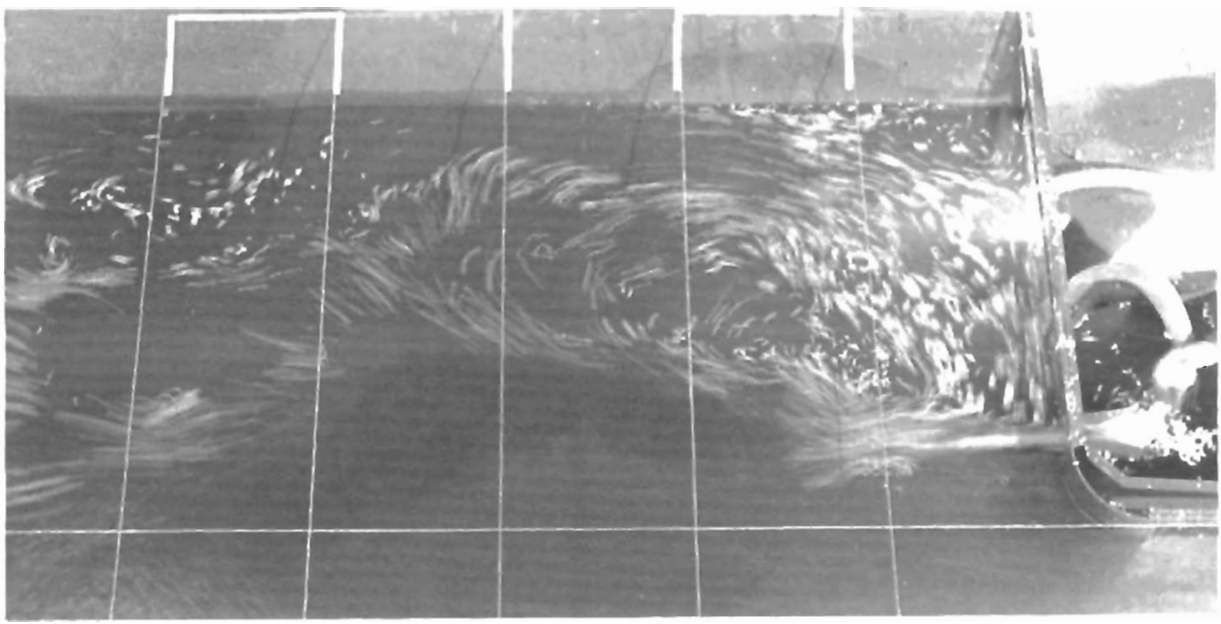

Photo $n^{\circ} 1$. - Ecoulement provenant de l'entrée principale de l'ascenseur débouchant dans la partie élargie du canal de fuite (configuration III sur figure 3)

Photo $\mathrm{n}^{\circ}$ 1. - Flow patterns at main fish elevator entrance (configuration III figure 3)

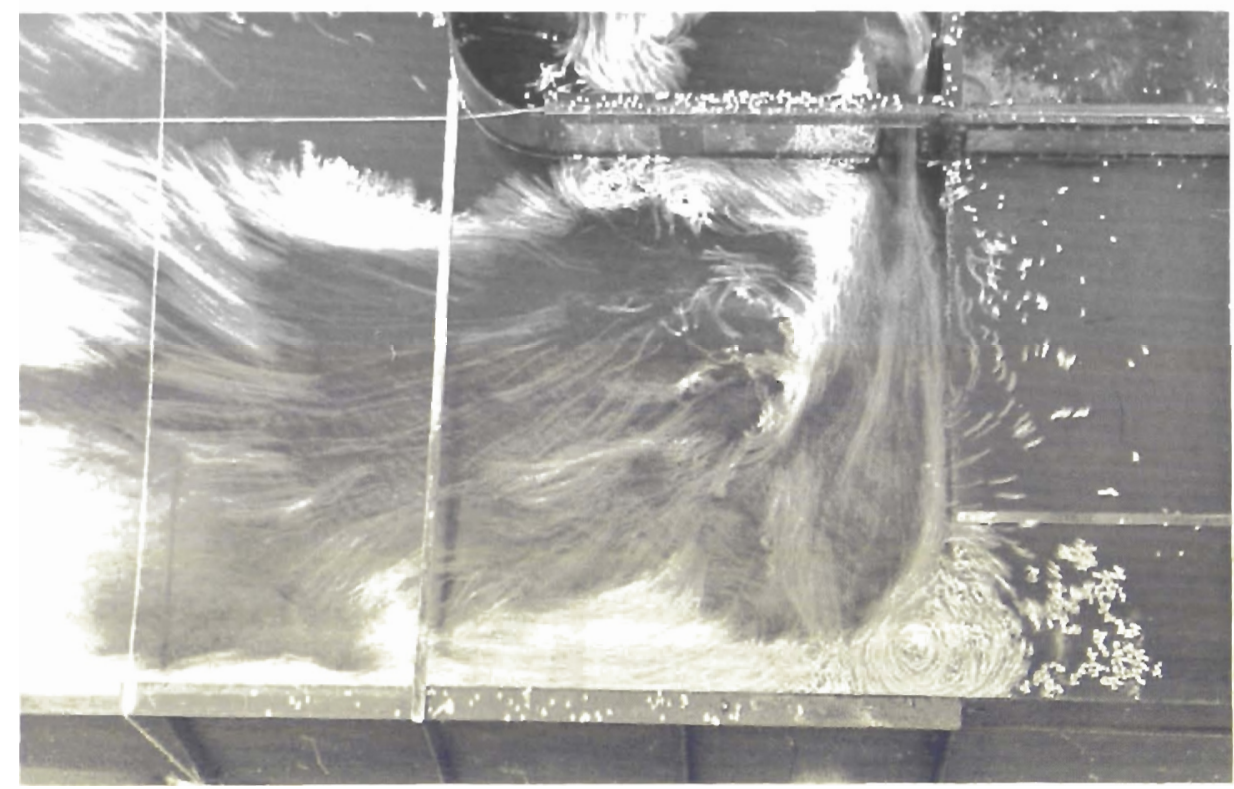

Photo $n^{\circ}$ 2. - Ecoulement de l'entrée secondaire située près de la sortie des turbines (configuration II sur figure 3)

Photo $\mathrm{n}^{\circ}$ 2. - Flow patterns at secondary entrance located near turbines outflow (configuration II figure 3) 


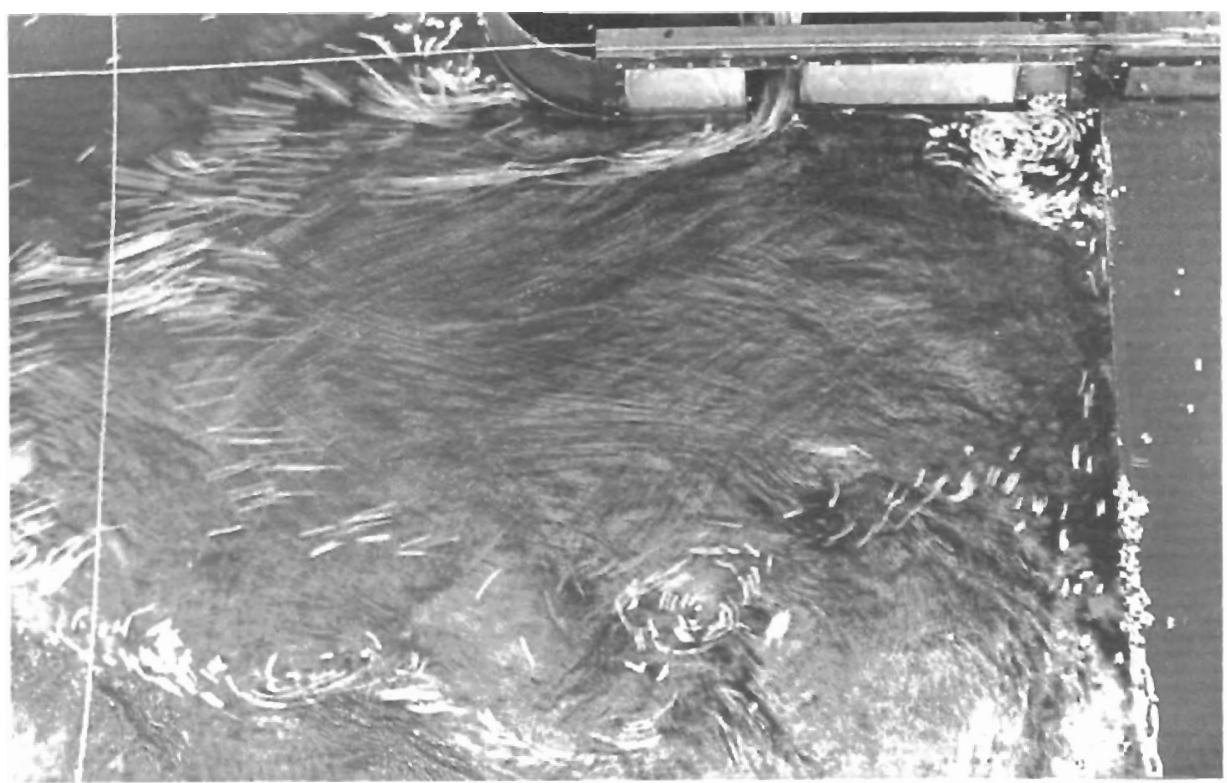

Photo $n^{\circ} 3 .-$ Ecoulement de l'entrée secondaire située dans la partie centrale du bajoyer rive droite à l'aval des turbines (configuration I figure 3 )

Photo $n^{\circ} 3$. - Flow patterns at secondary entrance located downstream turbines outflow (configuration I figure 3)

tablant sur une durée minimale de cycle (intervalle de temps séparant deux remontées de cuve) de 10 minutes, le volume de la cuve a été fixé à $5 \mathrm{~m}^{3}$. Pour des raisons constructives, ce volume a été réduit à $3,3 \mathrm{~m}^{3}$ mais pourrait être augmenté si le besoin s'en faisait sentir.

\section{6. - Description de l'ouvrage}

L'ascenseur se compose d'une partie basse (fig. 4 et 5 , photos 4 et 6 ) assurant l'attraction, la capture et la stabulation des poissons, d'une tour supportant le dispositif de relevage de la cuve et d'un canal de transfert assurant le transit des poissons vers le canal d'amenée.

\section{La partie basse comprend:}

- l'entrée du dispositif, pertuis de $1,7 \mathrm{~m}$ de largeur équipé d'une vanne déversante automatisée permettant de conserver un dénivelé constant entre le plan d'eau du bassin de stabulation et celui du bief aval,

- le bassin de stabulation d'une longueur de $9 \mathrm{~m}$, d'une largeur de $2,5 \mathrm{~m}$ et d'une profondeur variant de $1,5 \mathrm{~m}$ à $4,5 \mathrm{~m}$ suivant le débit dans la Garonne. L'alimentation en eau de ce bassin s'effectue au travers de grilles verticales situées à l'amont de la cuve et sur les parois latérales. Ces 


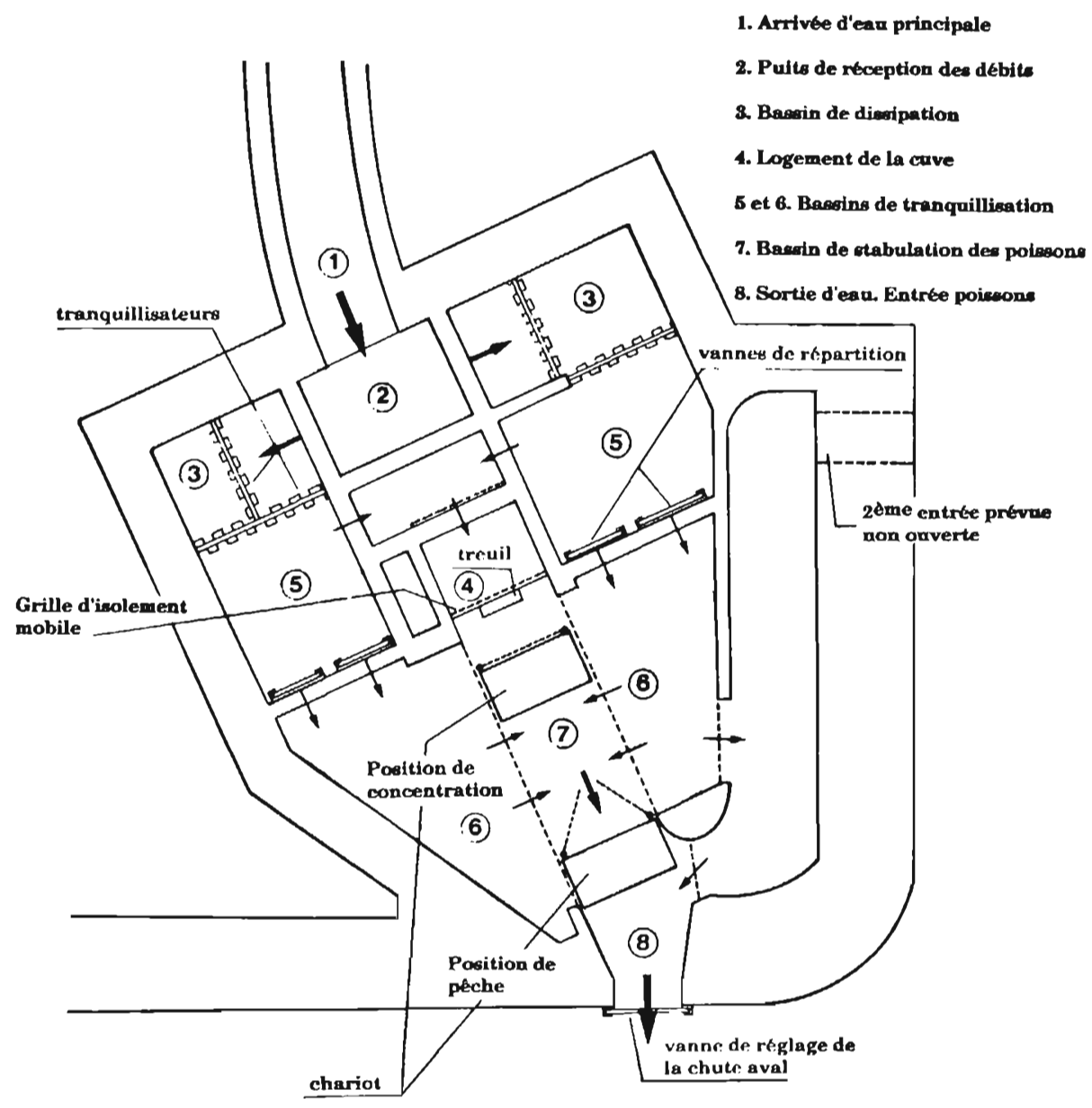

Fig. 4. - Schéma de la partie basse de l'ascenseur (vue en plan) : alimentation en eau, bassin de capture et de stabulation des poissons

Fig. 4. - Lower part of the fish elevator (plan view), water supply, capture and holding pool

grilles sont formées de barreaux de section circulaire (diamètre $1 \mathrm{~cm}$ ) espacées de $2,5 \mathrm{~cm}$,

- la fosse $(2,5 \mathrm{~m} \times 2,0 \mathrm{~m} \times 2,0 \mathrm{~m})$ dans laquelle vient reposer la cuve de l'ascenseur,

- des dispositifs de dissipation d'énergie et d'alimentation en eau du bassin de stabulation.
L'alimentation en eau du dispositif s'effectue gravitairement à partir du bief amont par l'intermédiaire d'un ancien canal d'évacuation des corps dérivants. La régulation de débit est assurée par un clapet de $2 \mathrm{~m}$ de largeur. Ce débit est filtré par une grille de $2,5 \mathrm{~cm}$ d'espacement dont le nettoyage est assuré par un dégrilleur automatique. 


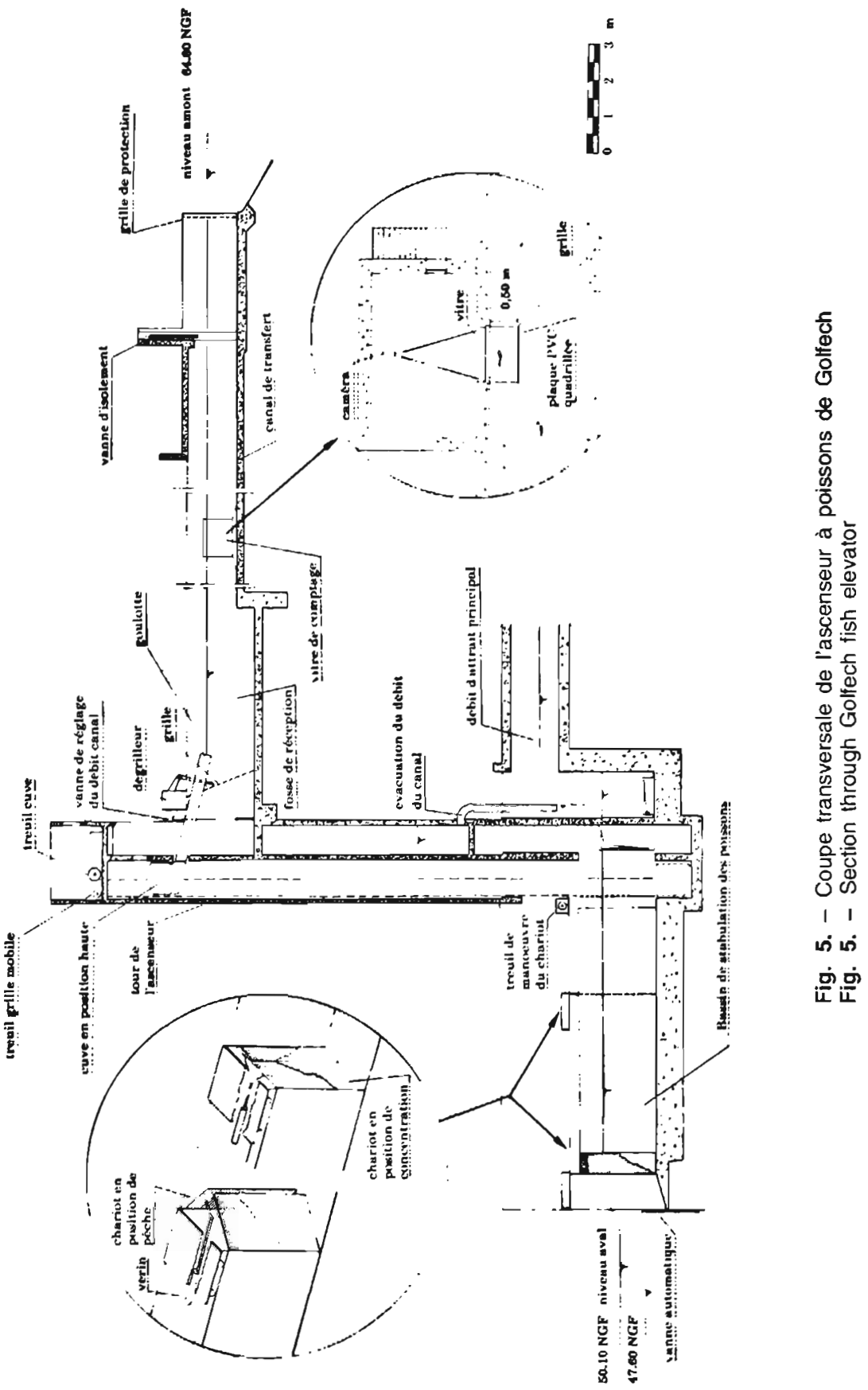




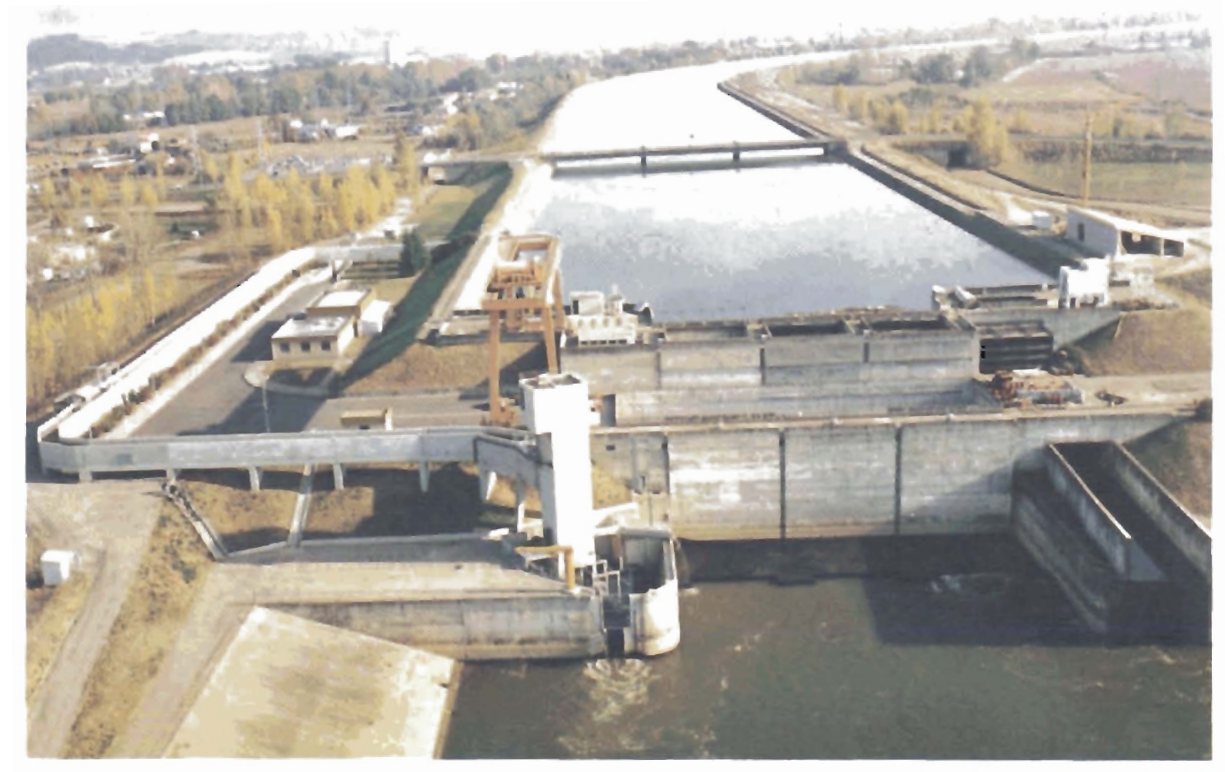

Photo $n^{\circ}$ 4. - Vue générale de l'usine de Golfech el de l'ascenseur à poissons Photo $n^{0} 4$. - General view of Golfech power plant and fish elevator

L'écran mobile se déplaçant dans le bassin de stabulation assure à la fois le rôle de nasse anti-retour et de concentration des poissons au-dessus de la cuve (fig. 5). II est constitué de deux panneaux de grilles $(1,5 \mathrm{~m} \times 4,5 \mathrm{~m})$ à barreaux horizontaux espacés de $2,5 \mathrm{~cm}$. Un vérin et un treuil hydrauliques permettent respectivement la fermeture de la nasse et la translation du chariot (photos 7 , 8,9 et 10 ).

La cuve, d'un volume de $3,3 \mathrm{~m}^{3}$ est remontée à l'aide d'un treuil hydraulique dans une tour en béton de 26 $\mathrm{m}$ de hauteur. Le déversement des poissons s'effectue par une trappe automatisée située en partie basse de la cuve (photo 11).
Le canal de transfert amont, de $250 \mathrm{~m}$ de longueur, d'une largeur de $2 \mathrm{~m}$ et d'une profondeur de $2,5 \mathrm{~m}$, permet de faire déboucher les poissons dans le canal d'amenée en amont de la zone de recirculation située au droit de la prise d'eau des turbines, cela afin d'éviter tout risque de désorientation des migrateurs à leur sortie du canal. Un débit maximum de $1 \mathrm{~m}^{3} / \mathrm{s}$ environ assure des vitesses suffisantes incitant les migrateurs à remonter à l'amont $(0,3 \mathrm{~m} / \mathrm{s}<\mathrm{V}<0,6 \mathrm{~m} / \mathrm{s})$.

Le fonctionnement de l'ouvrage, entièrement automatique, est régulé par deux automates programmables (S.M.C) assurant, pour l'un les fonctions mécaniques (mouvements de la 


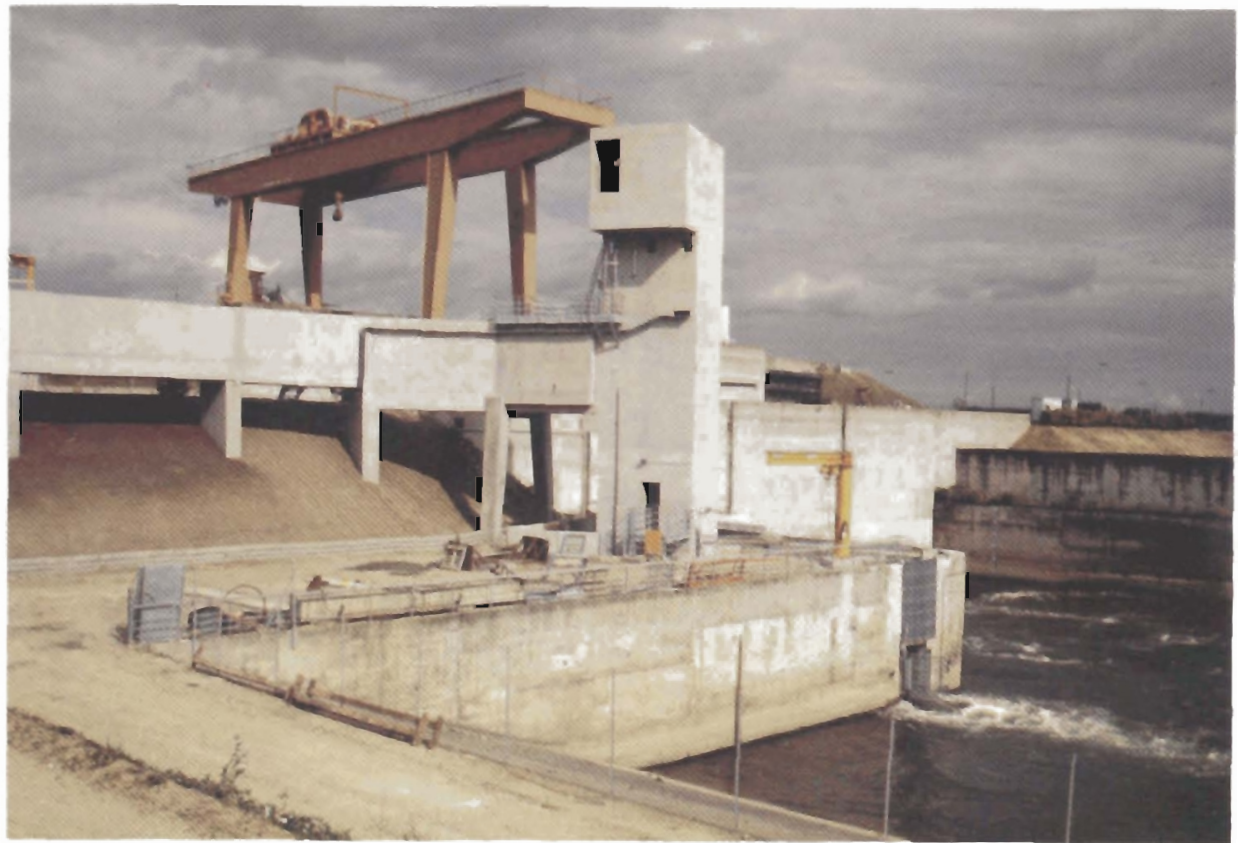

Photo $n^{\circ} 5$. - Entrée de l'ascenseur à poissons vue de l'aval

Photo $n^{\circ} 5$. - Downstream view of the fish elevator entrance

grille de capture et de concentration des poissons, levage et déversement de la cuve...) et pour l'autre, la régulation de l'alimentation en eau, de la vitesse dans le canal de transfert et du dénivelé à l'entrée de la passe. Tous ces paramètres sont modifiables par affichage de consignes.

La fréquence des remontées est réglable, la durée minimale entre deux cycles étant de $10 \mathrm{mn}$.

Une station de comptage visuel des poissons (fig. 2 et 4 , photos 12 et 13) est implantée sur le canal de transfert amont. Elle comprend une baie vitrée (largeur $1,5 \mathrm{~m}$, hauteur 2 $\mathrm{m})$ placée sur une paroi du canal et des grilles installées dans le canal obligeant les poissons à passer à proximité de cette baie vitrée (distance $\leq 50 \mathrm{~cm}$ ). Un éclairage de la zone de comptage est assuré pendant la nuit pour pouvoir effectuer les comptages 24 heures sur 24.

\section{7. - CONSTRUCTION DE L'OUVRAGE - COUT}

Du fait de l'absence d'expérience française en matière de passes à aloses et des inconnues subsistant quant à l'attractivité du dispositif, la construction de l'ouvrage s'est échelonnée sur trois années avec contrôle 


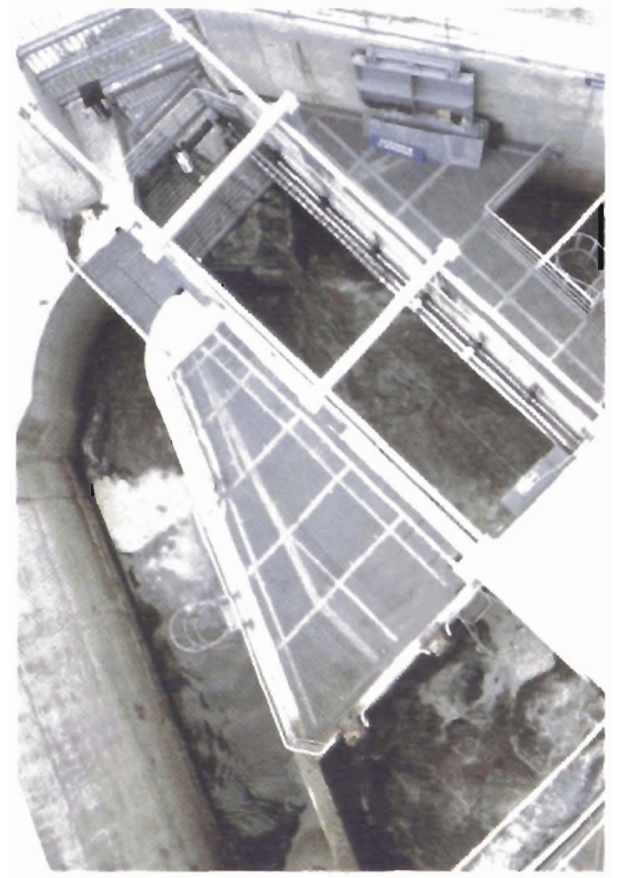

Photo $n^{\circ} 6$. - Vue d'ensemble de la partie basse de l'ascenseur: entrée, bassin de capture et stabulation, dispositifs de dissipation d'énergie et d'alimentention en eau (cf. fig. 4)

Photo $n^{\circ} 6$. - General view of the lower part of the elevator: entrance, holding pool, energy dissipation pool

de son efficacité à chacune des phases:

- phase 1 (1984-1985) : construction de la partie aval, c'est-à-dire du dispositif d'attrait et de stabulation. La capture de 3000 aloses dans le bas$\sin$ de stabulation à l'aide d'un système de piégeage provisoire a permis de démontrer l'attractivité de l'installation (Belaud et al. 1985),

- phase 2 (1986): construction du système d'attraction et de piégeage définitif comprenant les dispo- sitifs de régulation des vitesses de l'eau à l'entrée de la passe et la grille mobile de piégeage et concentration des poissons au dessus de la cuve. 6000 aloses ont été capturées (Bernard et Belaud 1986),

- phase 3 (1987): construction du dispositif de levage et du canal de transfert amont.

Le coût total de l'aménagement est de 23 millions de francs (valeur 1987).

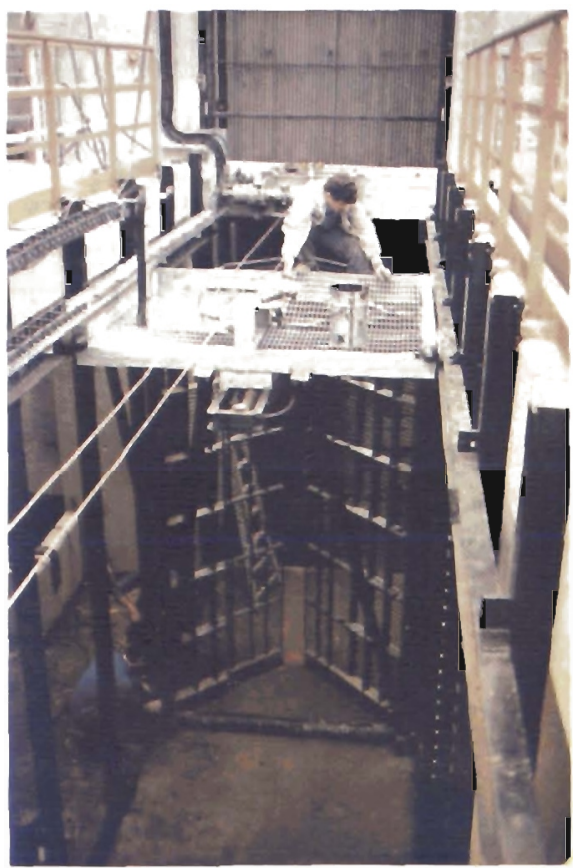

Photo $n^{\circ} 7$. - Grille mobile mécanisée assurant le rôle de piégeage (nasse anti-retour) et de concentration des poissons au dessus de la cuve

Photo $n^{\circ} 7$. - V-trap gates used as crowder when closed 


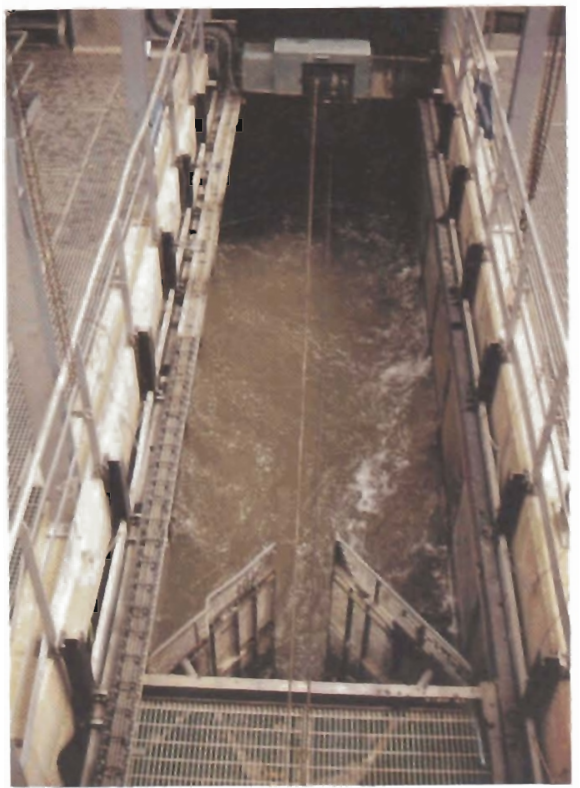

Photo $n^{\circ}$ 8. - Position du chariol mobile en phase de piégeage. Les grilles mécanisées sont ouvertes, formant nasse anti-retour (phase A) Photo $n^{\circ} 8$. - Trapping position of the V-trap gates (phase B)

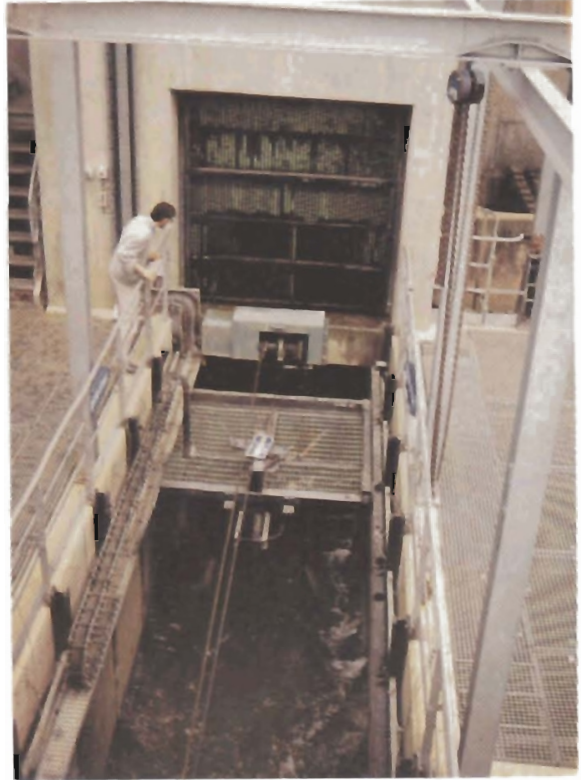

Photo $n^{\circ} 10$. - Chariot mobile en fin de phase de concentration des poissons. Descente de la grille mobile empêchant l'accès à lá losse de la cuve durant la manœuvre de cette dernière. (phase C)

Photo $n^{\circ}$ 10. - Crowder at the upstream part of the holding pool (phase C)

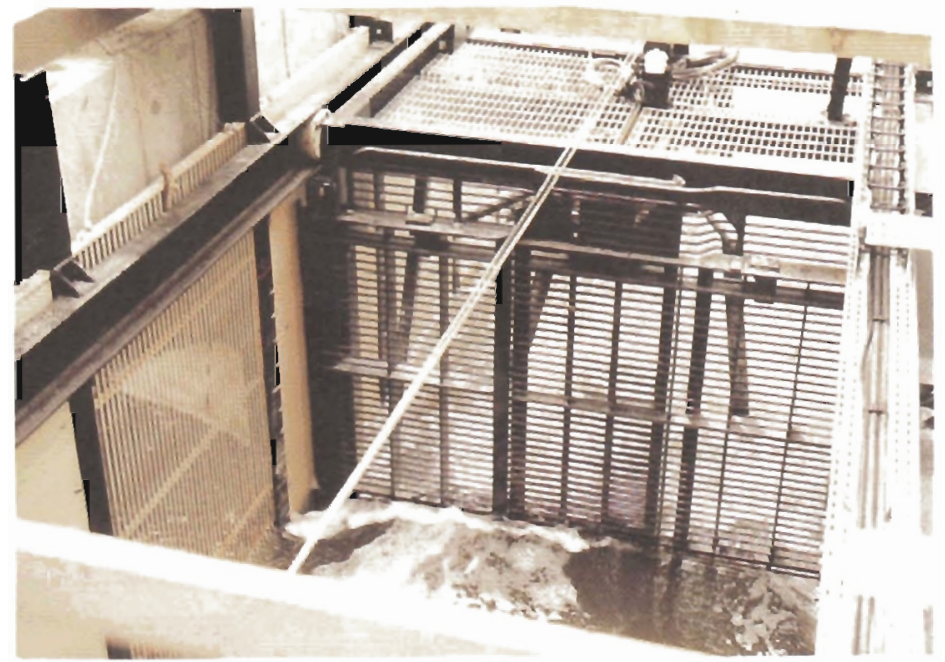

Photo $n^{\circ}$ 9. - Fermeture de la grille mécanisée pour la phase de concentration des poissons (phase B)

Photo $n^{\circ}$ 9. - Crowding position of the gates (phase B) 


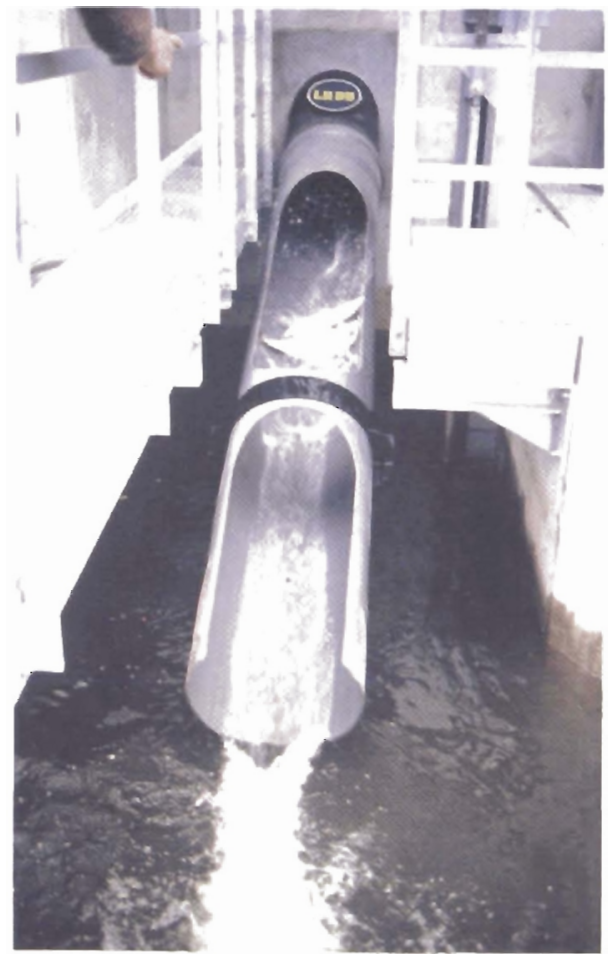

Photo $\mathrm{n}^{\circ} 11$. - Déversement de la cuve de l'ascenseur dans le canal de transfert.

Photo $n^{\circ} 11$. - Discharge of the hopper in the upper canal.

\section{8. - PREMIERS RÉSULTATS}

\subsection{Mise en route du dispositif}

L'automatisation très poussée du fonctionnement de l'ascenseur, et notamment des paramètres hydrauliques (chute à l'entrée, vitesse dans le bassin de stabulation et le canal de transfert, débit...) conditionnant son efficacité, a nécessité de nombreux réglages et modifications au cours des deux premières années faisant suite à la mise en service (Mai 1987).
Le seul problème subsistant à l'heure actuelle, résulte de l'ajustement périodique aux consignes de certains des paramètres de fonctionnement (vitesse dans le canal de transfert, débit principal dans l'ouvrage, chute à l'entrée...) qui génère des séquences de rattrapage de l'ensemble des autres paramètres. Elles se traduisent par des fluctuations quasi permanentes des conditions hydrauliques dans l'ouvrage qui se sont avérées très perturbantes pour les migrateurs et pénalisantes pour l'efficacité du dispositif. Une automatisation moins poussée de certains des paramètres (vitesse dans le canal de transfert par exemple) aurait permis une plus grande stabilité des conditions hydrauliques tout en assurant une meilleure fiabilité à l'installation.

Par ailleurs, le dispositif, compte tenu de la présence de nombreuses grilles, s'est révélé être très sensibie au problème de colmatage, en particulier lors des crues, ce qui entraîne des contraintes de maintenance beaucoup plus lourdes que celles afférentes à une passe classique.

\subsection{Déroulement du suivi-méthode}

L'insíallation a fait l'objet, de mai à décembre 1987 et 1988, de deux études de suivi destinées à l'optimisation de son fonctionnement mécanique et hydraulique et au contrôle de son efficacité biologique (Dartiguelongue, 1987, 1988). Diverses observations et expérimentations ont été conduites pour adapter aux principales espèces 
Ces opérations ont nécessité une quantification précise des rythmes de passages des poissons qui a été obtenue à l'aide d'un comptage visuel assisté par vidéo (système CERBERE) (Travade 1990). II consiste, dans son principe, à enregistrer sur magnétoscope uniquement les séquences de passage des poissons devant la baie vitrée de visualisation (fig. 5, photos 12 et 13). A cet effet, le magnétoscope est mis en route après détection des passages de poissons devant la vitre à l'aide d'un analyseur d'images. L'insertion d'une horloge sur l'enregistrement vidéo permet de repérer les heures de passage des poissons. Ce système a permis de disposer de chroniques de passage des poissons 24 heures sur 24.

\subsection{Optimisation des paramètres de fonctionnement}

Les expérimentations et observations ont permis de définir les valeurs optimales d'un certain nombre de paramètres de fonctionnement du dispositif. Les études ont été conduites principalement sur l'alose, espèce la plus exigeante vis à vis des conditions hydrodynamiques dans l'installation, et dont l'abondance permettait de mettre en œuvre un plan d'expérience. Les principaux résultats sont les suivants :

- le débit de l'ascenseur est réglé selon le niveau aval : il est voisin de $5,5 \mathrm{~m}^{3} / \mathrm{s}$ en temps normal mais inférieur à $3 \mathrm{~m}^{3} / \mathrm{s}$ en période de basses eaux, afin d'éviter des vitesses trop élevées dans le bassin de stabulation. Ces débits représentent de $1 \%$ à $5 \%$ du débit turbiné à l'usine,

- la chute optimale à l'entrée du piège est voisine de $25 \mathrm{~cm}$ (vitesse voisine de $2,2 \mathrm{~m} / \mathrm{s}$ ) en période de présence des aloses. Il est difficile de savoir si cet optimum est uniquement lié au comportement de l'alose et dans quelle mesure il est influencé par la configuration du site (nature des écoulements au voisinage de l'entrée),

- les vitesses au droit de la fente d'entrée dans la nasse anti-retour, nécessaires pour inciter les poissons à pénétrer dans le bassin de stabulation sont de l'ordre de $0,6 \mathrm{~m} / \mathrm{s}$ à $1 \mathrm{~m} / \mathrm{s}$,

- l'ouverture de la nasse, empêchant l'échappement des poissons vers l'aval tout en ne faisant pas obstacle à leur entrée, est de l'ordre de $0.40 \mathrm{~m}$,

- la durée des cycles, réglée seIon l'abondance des poissons varie de 1-2 heures à un minimum de 10 minutes en période de pic de migration de l'alose,

- la vitesse de l'eau dans le canal de transfert doit être au minimum de $20 \mathrm{~cm} / \mathrm{s}$ à $25 \mathrm{~cm} / \mathrm{s}$, vitesse en deçà de laquelle le comportement des principaux poissons migrateurs est perturbé.

Des résultats plus qualitatifs ont été obtenus sur d'autres espèces comme l'anguille, pour laquelle la chute optimale à l'entrée du dispositif est voisine de $5 \mathrm{~cm}$. 


\subsection{Mortalité des poissons}

Le fait de concentrer un grand nombre d'individus dans des espaces réduits (bassin de stabulation, cuve, goulotte de vidange de la cuve, canal de transfert) et de les forcer à cotoyer des structures métalliques mobiles (portes, chariot, grilles ou cuve) entraîne inévitablement des risques de blessures et de mortalités.

En 1988, on s'est attaché à apprécier l'importance des dommages. L'alose s'avère être l'espèce la plus sensible. Le pourcentage de mortalité pour l'alose reste néanmoins faible: $0,5 \%$ de la population ayant emprunté le dispositif. Cette valeur est du même ordre de grandeur que celle observée sur l'alose américaine à l'ascenseur de Holyoke (Rizzo, comm.pers.). Ces chiffres ne prennent évidemment pas en compte une éventuelle mortalité différée qui reste difficilement appréciable.

Les blessures d'origine mécaniques (sectionnement, décapitation, écrasement...) sont prédominantes; il est probable qu'elles se produisent lors de la phase de remontée de la cuve. Aucune mortalité massive attribuable à une abondance excessive de poissons dans la cuve n'a été constatée lors des pointes de passage où le nombre maximal d'aloses a dépassé à plusieurs reprise 300 individus par remontée. Cette quantité d'aloses correspond à un volume d'eau dans la cuve de 11 litres par alose, ce qui encore en dessous du seuil critique adopté lors du dimen- sionnement (5,7 litres par alose) (Dalley, 1980), mais très voisin du seuil utilisé actuellement aux USA de 10 litres par alose (Rizzo, 1986). Si dans l'avenir, les passages augmentaient de façon significative, il est vraisemblable qu'un accroissement du volume de la cuve s'avèrera nécessaire.

L'autre source notable de mortalité est le coincement des poissons entre les barreaux des grilles d'injection du débit dans le bassin de stabulation. A cet égard, il semblerait que des barreaux de section rectangulaire ou des grilles à mailles rectangulaires soient préférables aux grilles à barreaux de section circulaires utilisées à Golfech. L'utilisation de grilles à mailles rectangulaire entraîne cependant des contraintes d'entretien beaucoup plus importantes.

\subsection{Quantification et caractéristi- ques des passages de poissons}

\subsubsection{Bilan général des passages de poissons}

Environ 25 espèces ont emprunté le dispositif dont 5 espèces amphibiotiques (saumon, truite de mer, alose, lamproie, anguille) représentant plusieurs dizaines de milliers d'individus (tableau I).

Parmi les grands migrateurs, l'espèce la plus abondante est l'alose (18000 et 14000 en 1987 et 1988) et respectivement $66000,45000^{(2)}$ et 40000 pour 1989, 1990 et 1991, (ENSAT 1989, 1990). Les passages d'anguille (présente sur le site sous forme

(2) Estimation par échantillonnage. 
Tableau I. - Passage des différentes espèces à l'ascenseur de Golfech de 1987 à 1989. Table I. - Fish passages at Golfech elevator from 1987 to 1989

\begin{tabular}{|c|c|c|c|c|}
\hline NOM COMMUN & NOM SCIENTIFIOUE & EFFECTIF 1987 & EFFECTIF 1988 & EFFECTIF 1989 \\
\hline $\begin{array}{l}\text { MIGRATEURS AMPHIBIOTIQUES } \\
\text { Alose } \\
\text { Anguille } \\
\text { Lamproie marine } \\
\text { Saumon atlantique } \\
\text { Truite de mer } \\
\text { Salmonidés indet. } \\
\quad \text { AUTRES ESPECES } \\
\text { Ablette } \\
\text { Barbeau } \\
\text { Blackbass } \\
\text { Brème } \\
\text { Breme bordeliere } \\
\text { Brochet } \\
\text { Carassin } \\
\text { Carpe } \\
\text { Chevesne } \\
\text { Gardon } \\
\text { Muge } \\
\text { Perche } \\
\text { Perche soleil } \\
\text { Poisson chat } \\
\text { Rotengle } \\
\text { Sandre } \\
\text { Tanche } \\
\text { Truites de riviere }\end{array}$ & $\begin{array}{l}\text { Aosa alosa } \\
\text { Anguilla anguilla } \\
\text { Petromyzon marinus } \\
\text { Salmo salar (a) } \\
\text { Salmo trutta trutta (b) } \\
\text { (a) ou (b) } \\
\\
\text { Alburnus alburnus } \\
\text { Barbus barbus } \\
\text { Micropterus salmoides } \\
\text { Abramis brama } \\
\text { Blicca bjorkns } \\
\text { Esox lucius } \\
\text { Carassius auratus } \\
\text { Cyprinus carpio } \\
\text { Leuciscus cephalus } \\
\text { Autilus rutilus } \\
\text { Mugil capito } \\
\text { Perca lluviatills } \\
\text { Lepomis gibbosus } \\
\text { Ictalurus melas } \\
\text { Scardinus orythrophtalmus } \\
\text { Stizostedion lucioperca } \\
\text { Tinca linca } \\
\text { S. trutta fario } \\
\text { S. gairdneri }\end{array}$ & $\begin{array}{c}18224 \\
4970(1) \\
11 \\
24 \\
51 \\
22 \\
\\
217(1)(2) \\
2356 \\
13 \\
3256 \\
\text { presence } \\
2 \\
1 \\
9 \\
6 \\
\text { présence } \\
0 \\
7 \\
5 \\
3433 \\
\text { presence } \\
90 \\
2 \\
32 \\
\end{array}$ & $\begin{array}{c}13779 \\
1166(1) \\
16 \\
25 \\
48 \\
18 \\
\\
18800(1) \\
1048 \\
9 \\
3830 \\
\text { presence } \\
4 \\
6 \\
32 \\
3 \\
\text { pressence } \\
1583 \\
1 \\
9 \\
2908 \\
\text { présence } \\
572 \\
2 \\
17 \\
\end{array}$ & $\begin{array}{c}66401 \\
13322(1) \\
370 \\
1 \\
5 \\
\\
1003(1)(2) \\
3284 \\
10743 \\
\text { presence } \\
6 \\
\text { pressence } \\
20 \\
50 \\
\text { presence } \\
404 \\
4 \\
2 \\
1486 \\
\text { presence } \\
250 \\
2 \\
29 \\
\end{array}$ \\
\hline
\end{tabular}

(1) : nombre sous-estimé

(2) : nombre de bancs

d'anguillette d'une vingtaine de centimètres de longueur), sont limités à quelques milliers d'individus, mais sous-estimés du fait de l'efficacité partielle du dispositif de comptage pour cette espèce. La lamproie marine est en très faible nombre (quelques dizaines à quelques centaines d'individus). II en va de même pour les grands salmonidés (saumon et truite de mer) dont les effectifs sont limités à quelques dizaines d'individus, ce qui s'explique par la récence du plan de restauration de ces espèces sur la Garonne.
Parmi les autres familles et espèces de poissons empruntant le dispositif, les cyprinidés sont la plus abondante avec plusieurs milliers d'individus répartis en une dizaine d'espèces. Le barbeau, l'ablette et la brème (ou espèces non discernables à la vidéo : brème bordeliere, gardon, rotengle) sont les plus représentés.

\subsubsection{Caractéristiques des pas- sages}

Les données biologiques recueillies lors du suivi des ouvrages de franchissement peuvent permettre d'accéder à une meilleure connais- 


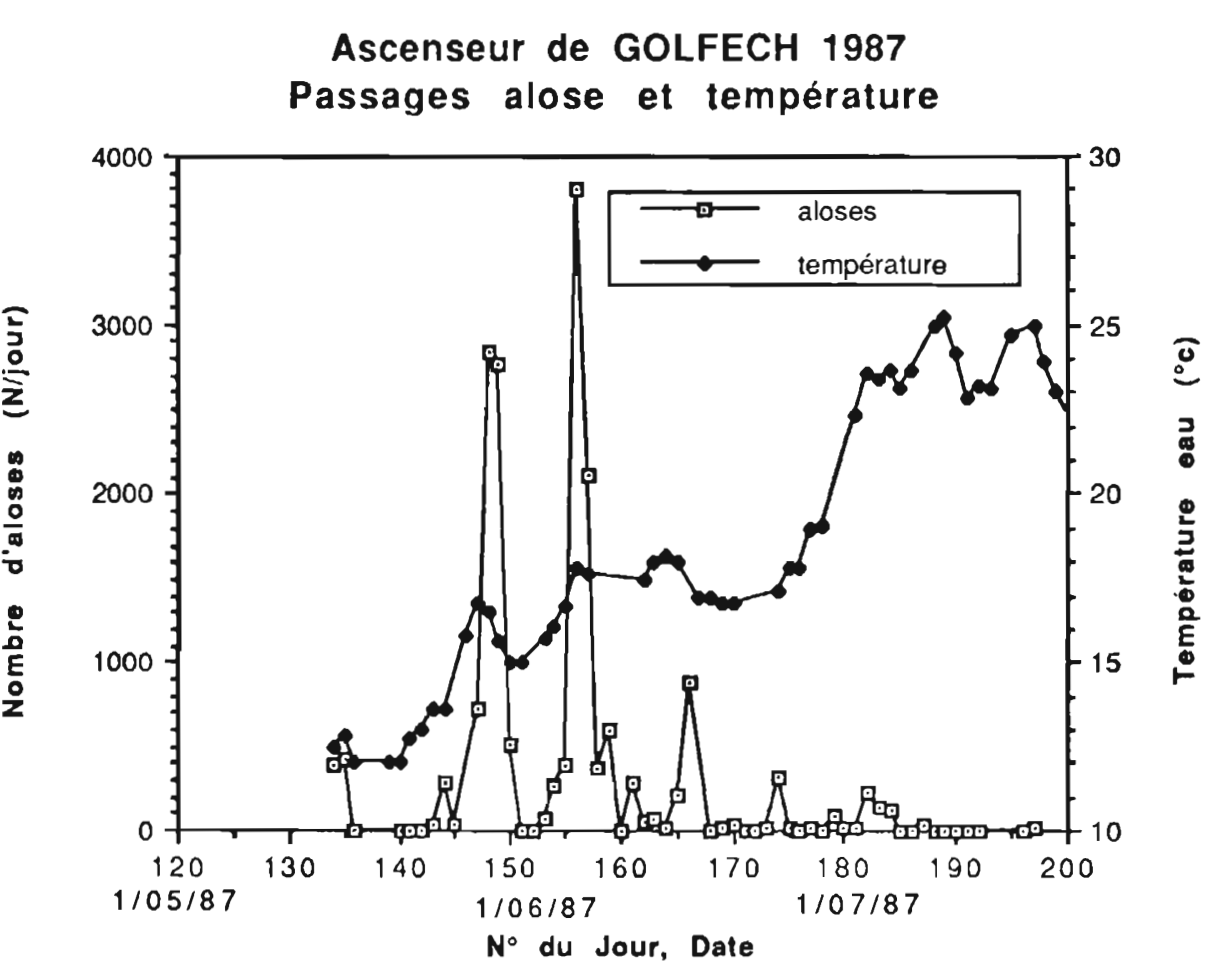

Fig. 6. - Passages journaliers d'aloses en 1987 à l'ascenseur et effets de la température de l'eau Fig. 6. - Daily shad passages at Golfech elevator in 1987. Water temperature effect.

sance des caractéristiques de migration des principales espèces: calendrier, effectif, amplitude du pic migratoire ou rythme nycthéméral. Leur prise en compte s'avère nécessaire à l'optimisation des critères de dimensionnement (volume du bassin de stabulation et de la cuve...) et des paramètres de fonctionnement du dispositif (adaptation aux différentes espèces suivant leurs heures ou périodes de passage...).

Bien que la représentativité de tels résultats repose sur l'obtention de séries chronologiques complètes et suffisamment longues, les premières années de suivi ont permis de mettre en évidence quelques caractéristiques importantes des rythmes migratoires sur le site de Golfech.

\section{- Rythme d'activité saisonnière}

Les suivis effectués se sont déroulés de mai à juillet et d'octobre à décembre englobant les périodes de migration des principales espèces amphibiotiques et de déplacement de la majorité des espèces holobiotiques.

II n'est cependant pas certain que la totalité de la migration ait été prise en compte pour certaines espèces 
Tableau II. - Caractéristiques des pics de migration de l'alose à Golfech de 1987 à 1991 Table II. - Characteristics of the maximum daily passages of shad at Golfech elevator from 1987 to 1991.

\begin{tabular}{|l|c|c|c|c|c|}
\hline & 1987 & 1988 & 1989 & 1990 & 1991 \\
\hline Passage annuel (nb) & 18000 & 13000 & 66000 & 45000 & 40000 \\
\hline Pic journalier & & & & & \\
Date & & & & \\
Temperature ('C) & $5 / 06$ & $26 / 05$ & $12 / 06$ & $13 / 06 / 30 / 06$ & $22 / 06$ \\
Passage (nb) & 17.3 & 15.4 & 20.3 & $19.0 / 23.5$ & 18.5 \\
* passage annuel & 3800 & 1700 & 4500 & $5000 / 5900$ & 3400 \\
& $21 \times$ & $13 \times$ & $7 *$ & $11 \% / 13 \times$ & $8.5 *$ \\
\hline
\end{tabular}

précoces telles que la lamproie, le brochet ou le sandre dont l'activité peut débuter en mars-avril, ou pour les salmonidés dont la migration peut s'étaler tout au long de l'année.

Pour les grands migrateurs, la distribution saisonnière des passages peut être soit unimodale de printemps-été (alose, lamproie, anguille), soit bimodale avec un pic de printemps et un pic d'automne (salmonidés).

Les passages d'alose confirment les informations recueillies de 1980 à 1986 lors des opérations de transfert manuel antérieures à la mise en service de l'ascenseur (Belaud et Labat, 1991), à savoir une migration de deux mois environ située dans la période avril-juillet, se présentant sous forme d'une succession de pics influencés par la température de l'eau (fig. 6). Ces pics journaliers de migration (tableau II) sont du même ordre de grandeur $(7 \%$ à $21 \%$ du passage annuel de 1987 à 1991) que ceux observés lors des opérations de capture $(4 \%$ à $17 \%$ ). La valeur élevée de $21 \%$ ob- servée en 1987 est probablement liée au fonctionnement irrégulier de l'ascenseur alors en cours de mise au point.

La migration de l'anguille se situe en fin de printemps et début d'été (juin à août). Elle présente des pics de passage similaires à ceux de l'alose.

La lamproie marine migre plus précocement (avril-mai). Le faible nombre d'individus observé ne permet pas de conclure quant à la nature de la migration. II est possible par ailleurs qu'une partie de la migration ait été tronquée par le démarrage tardif du suivi.

Les salmonidés présentent deux périodes de migration, l'une printanière (mai-juillet), l'autre automnale (octobre-décembre). Les passages sont encore trop faibles pour déterminer l'importance respective des deux vagues migratoires.

Pour les espèces holobiotiques, on observe de façon générale une période d'activité printanière (avril-juillet) qui peut être suivie d'une reprise d'activité automnale (octobre-décem- 


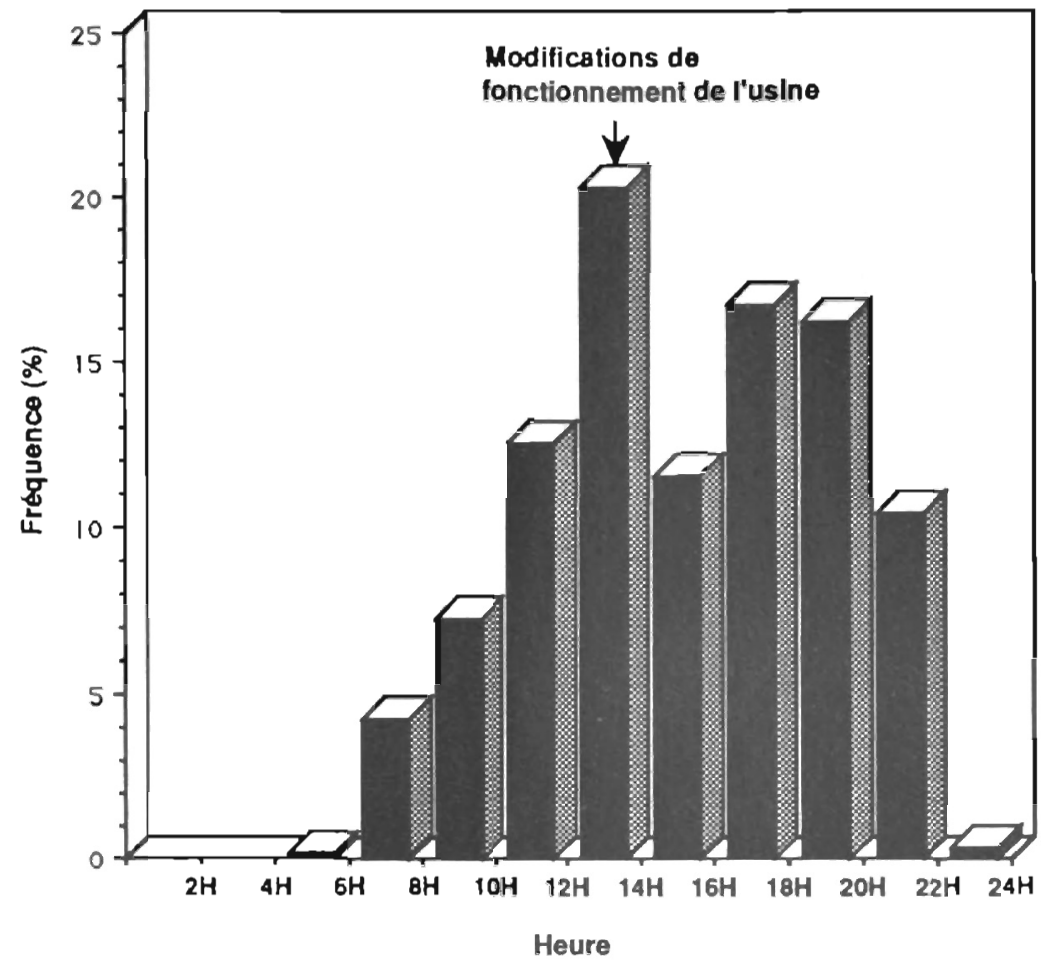

Fig. 7. - Passages horaires des aloses à l'ascenseur en 1988. Effel du fonctionnement de l'usine. Fig. 7. - Hourly shad passages at Golfech elevator in 1988. Effect of Power plant operation.

bre), de moindre ampleur, chez certaines espèces telles que le brochet, le sandre, le black-bass, le barbeau et la brême (ou espèces confondues à la vidéo).

\section{- Rythme d'activité journalière}

Les seules données accessibles sont celles obtenues à partir des enregistrements vidéo $24 \mathrm{~h}$ sur 24 effectuées à la vitre de comptage. Les rythmes obtenus ne coïncident pas avec ceux des entrées dans l'ouvrage. II existe en effet un laps de temps entre le moment où le poisson se présente à l'entrée de l'ascenseur et celui où il passe devant la vitre. Cette durée, difficile à évaluer, est vraisemblablement très variable en fonction du cycle de l'ascenseur (durée de la phase de piégeage), des conditions de milieu et des espèces (durée du transit dans le canal, hésitation avant le passage devant la vitre, perturbation par l'éclairage de la vitre de comptage...). Cependant, compte tenu de la brièveté des cycles (10 mn à $20 \mathrm{mn}$ ) au printemps, principale période de migration pour la grande majorité des espèces, les 
rythmes obtenus à la vitre peuvent être considérés comme représentatifs des rythmes naturels.

La plupart des espèces observées sur le site présentent un rythme d'activité à dominante diurne: c'est le cas de l'alose (fig. 7) et des salmonidés chez les migrateurs amphibiotiques, ou des cyprinidés pour les espèces holobiotiques. Les espèces dont la période d'activité est surtout nocturne sont essentiellement l'anguille, la lamproie ou le sandre. Chez l'anguille, on a noté cependant certains jours une activité diurne essentiellement en milieu de journée vers $12 h-13 h$.

Que ce soit pour les espèces diurnes ou nocturnes, on observe en général une ou plusieurs périodes d'activité plus intense au cours du nycthémère (de $13 \mathrm{~h}$ à $20 \mathrm{~h}$ chez l'alose, de $22 \mathrm{~h}$ à $6 \mathrm{~h}$ chez l'anguille...).

- Facteurs influençant les rythmes de migration et le passage dans l'ascenseur

Les rythmes saisonnier et journalier peuvent être influencés par des facteurs de milieu, naturels (température de l'eau, débit fluvial, photopériode) ou artificiels (régime de fonctionnement de l'usine). La température de l'eau ou le débit ont plutôt une action sur l'activité saisonnière. Cet effet est par exemple très net chez l'alose ou les cyprinidés pour lesquels on observe une recrudescence des mouvements pendant les périodes d'accroissement thermique et à l'inverse un ralentissement, voire un arrêt de l'activité suite à une chute de température (fig. 6). Le régime de fonctionnement de l'usine semble agir sur les passages dans l'ascenseur: les modifications brutales du fonctionnement des différents groupes (s'étant produites systématiquement entre $12 \mathrm{~h}$ et $14 \mathrm{~h}$ en 1988, fig. 7), entraînent une baisse des passages pour la plupart des espèces, notamment l'alose et les cyprinidés.

II est probable que la répartition du débit selon les différents groupes influe sur l'attractivité de l'ascenseur. Lorsque le groupe rive droite est en fonctionnement, on observe une meilleure stabilité du jet provenant de l'ascenseur et un rassemblement des aloses dans la zone proche de l'entrée de l'ouvrage. II n'a cependant pas été possible de mettre en évidence statistiquement l'influence de ce facteur sur les passages d'aloses à l'ascenseur, en raison de la trop grande variabilité de leur abondance naturelle au pied de l'usine.

\section{9. - DISCUSSION ET CONCLUSIONS}

Les principes de fonctionnement et les critères de dimensionnement de l'ascenseur construit à Golfech ont été directement inspirés de l'expérience nord-américaine, aucun ouvrage de ce type n'existant ni en France ni en Europe au moment du lancement du projet.

A l'expérience des premières années de suivi, il apparaît que, sur le plan technique, un ascenseur à poissons, compte tenu des nombreux automatismes qui assurent son fonctionnement, nécessite des ajuste- 
ments et une maintenance beaucoup plus importants que les autres types de passes à poissons. De ce fait, il est essentiel, lors de la conception d'un tel dispositif, de chercher à en rendre le fonctionnement le plus simple et rustique possible. D'autre part, la présence obligatoire d'un certain nombre de grilles se traduit par une sensibilité particulière aux problèmes de colmatage qui peuvent être en partie résolus par l'installation de systèmes de dégrillage efficaces mais induisent de toute façon des charges d'entretien conséquentes.

Sur le plan biologique, il s'avère que ce dispositif est peu sélectif, dans la mesure où la quasi-totalité des espèces présentes sur le site l'ont emprunté, notamment des espèces telle que l'alose, reconnue comme étant particulièrement exigeante vis à vis des dispositifs de franchissement, et le sandre, qui n'est que très rarement observé dans les passes à bassins.

II est cependant difficile, en l'absence de quantification des populations des principales espèces migratrices (saumon, truite de mer, alose, lamproie, anguille) à l'aval de l'ouvrage, ou d'expérimentations plus spécifiques (marquage, radiopistage...), d'évaluer l'efficacité réelle du dispositif pour chacune de ces espèces.

Il est probable que la grande majorité des salmonidés trouvent plus ou moins rapidement l'entrée du dispositif, sans qu'il soit possible de dire si les retards induits dans leur migration sont importants ou non.
Par contre, il est clair que l'efficacité du dispositif n'est que partielle pour l'alose, même si le nombre d'individus ayant emprunté le dispositif (de 14000 à 660000 individus par an) peut paraître important. L'observation, au pied de l'usine, de quantités de poissons visuellement beaucoup plus importantes que les remontees en sont un indice. L'ordre de grandeur de l'efficacité pour cette espèce, bien qu'impossible à préciser dans l'état actuel de nos connaissances, serait cependant de quelques dizaines de pour cent. II est certain que l'efficacité du dispositif aurait été significativement plus importante si la deuxième entrée prévue initialement, située à proximité immédiate du pied des turbines où se rassemblent de très nombreuses aloses, avait été ouverte.

D'autre part, pour l'anguille, l'efficacité de l'ascenseur n'est que très partielle (quelques pour mille à quelques pour cent?). Ceci est inhérent à la nature du dispositif, et notamment à l'inadaptation à cette espèce (ainsi qu'aux autres espèces de petite taille) du système de piégeage et de concentration des poissons au dessus de la cuve. Du fait de la porosité des grilles, supérieure à la taille des anguillettes, la capture ne se fait qu'aléatoirement pour les individus nageant au dessus de la cuve au moment de sa remontée. L'observation d'accumulations d'anguilles dans l'ouvrage de capture corrobore cette conclusion. II est illusoire d'envisager raisonnablement la diminution de la porosité des grilles de façon à captu- 
rer ces petites espèces, car cela entraînerait inévitablement des problèmes d'entretien et de maintenance insolubles.

Le retour d'expérience, acquis par le suivi biologique et technique de l'ascenseur a déjà permis, au bout de quelques années, une reflexion sur la pertinence et l'importance des différents critères de dimensionnement pris en compte au départ du projet, et alors issus de l'expérience étrangère. On constate par exemple, que les pics journaliers de passages d'aloses $(7 \%$ à $13 \%$ de 1988 à 1991) sont compatibles avec le principe de dimensionnement de l'ouvrage prenant en compte un passage journalier de $10 \%$ de la population. Certains critères (débit d'attraction, vitesses d'écoulement, chutes...) ont pu être adaptés aux espèces migratrices françaises, d'autres méritent d'être encore affinés et nécessitent une poursuite des contrôles sur ce type d'ouvrage.

Les premiers résultats acquis sur les rythmes et les comportements migratoires permettent dès à présent de mieux gérer le dispositif. En particulier, le calendrier d'arrivée sur le site des principaux migrateurs détermine la période durant laquelle le dispositif doit être impérativement fonctionnel et efficace; a contrario, les périodes creuses dans l'année peuvent être mises à profit pour programmer les opérations d'entretien et de travaux. La connaissance des rythmes nycthéméraux et saisonniers peut permettre une gestion et des réglages adaptés à certaines espèces (abaissement de la chute à l'entrée du dispositif durant la nuit pour l'anguille...).

Sur un autre plan, l'ascenseur à poissons de Golfech, compte tenu de sa situation privilégiée et de ses équipements, s'avère être une station de contrôle des migrateurs particulièrement intéressante. II apparaît, à ce titre, comme un outil indispensable à la gestion à long terme des stocks de migrateurs de l'ensemble du bassin de la Garonne.

\section{RÉFÉRENCES}

Belaud A., Dautrey R., Labat R., Lartigue J.P., Lim P., 1985. Observations sur le comportement migratoire des aloses (Alosa alosa L.) dans le canal artificiel de l'usine de Golfech. Ann. Limnol., 21, (2), 161-172.

Belaud A., Labat R., 1984. Le comportement migratoire des aloses (Alosa alosa $L$.) dans le canal de restitution de l'usine de Golfech. Effet de la température. Ictyophysiologica acta 9, 177186.

Belaud A., Labat R., Trivellato D. Tison G., 1987. Expérimentations hydrauliques et ichtyologiques d'un système de piégeage automatique des poissons migrateurs à Golfech. La Houille Blanche $n^{\circ} 1 / 2,73-79$.

Belaud A., Labat R., 1991. Etudes ichtyologiques préalables à la conception d'un dispositif de tranchissement du barrage de Golfech (Garonne) par les poissons migrateurs. Hydroécologie appliquée, à paraître.

Bernard S., Belaud A., 1986. Transfent d'aloses 1986 à Golfech. Expérimentations du système de piégeage définitif. Rapport ENSAT, $30 \mathrm{p}$.

Bell M.C., 1973. Fisheries handbook of engineering requirements and biological criteria. Fisheries Engineering Research Program. U.S. Army Corps of 
Engineers, North Pacific Division. Portland, Oregon.

Cassou-Leins F., Cassou Leins J.J., 1980. Recherches sur la biologie et l'halieutique des migrateurs de la Garonne, et principalement de l'alose (Alosa alosa L.). Thèse doctorat $3^{e}$ cycle, INP Toulouse, $382 \mathrm{p}$.

Dalley P.J., 1980. A review of fish passage facilities for american shad. Northeast Fish and Wildlife Conference. Ellenville, New York, $32 \mathrm{p}$.

Dartiguelongue J., 1987. Suivi de la migration des aloses à Golfech en 1987. Expérimentations du dispositif de transfert des poissons. Rapport ATIA, $71 \mathrm{p}$.

Dartiguelongue J., 1988. Mise au point du dispositif de transfert des poissons à Golfech. Suivi de l'activité ichtyologique en 1988. Rapport ATIA, 57 p.

Douchement C., 1981. Etude des populations d'aloses, poissons migrateurs des fleuves français. Thèse doctorat $3^{a}$ cycle, Université des Sciences et Techniques du Languedoc, $167 \mathrm{p}$.

ENSAT, 1989. Centrale de Golfech : étude ichtyologique. Actualisation du point zéro. Rapport final, $57 \mathrm{p}$.

ENSAT-CSP, 1990. Recherches sur les aloses ayant franchi le complexe hydroélectrique de Golfech : fraie, développement des juvéniles, pêche. Saison 1990. Rapport final ENSATCSP, $38 \mathrm{p}$.

I.M.F.T., 1985. Aménagement hydraulique de Golfech. Franchissement par les poissons migrateurs, étude du débit d'attrait. Rapport n402.1, 105 p.

Larinier M., 1981. Migration des aloses dans le Bas Rhône. Observations effectuées en 1979 et 1980. Rapport interne CTGREF-DQEPP, Janvier 1981, $10 \mathrm{p}$.

Larinier M., 1983. Guide pour la conception des dispositifs de franchissement des barrages pour les poissons migrateurs. Bulletin Français de Pisciculture, numéro spécial, $39 \mathrm{p}$.
Larinier M., Travade F., 1982. Les poissons migrateurs aux Etats-Unis. Ouvrages de franchissement des barrages. Programmes de restauration des populations. EDF-CEMAGREF, 73 p. + annexes.

Larinier M., Trivellatto D., 1987. Utilisation des modèles réduits pour l'étude des dispositifs de franchissement sur les grands cours d'eau. In M. Thibault et R. Billard, Ed. Restauration des rivières à saumons. INRA, Paris, 149157.

Puyo C., Venel A., 1987. Contribution d'un modèle réduit physique aux études d'ingénierie de l'ascenseur à poissons de Golfech. La Houille Blanche, $n^{\circ} 1 / 2$, 81-87.

Rideout S., Thorpe L., Cameron L.,1985. Passage of american shad in an Ice Harbor style fish ladder after flow pattern modifications. Symposium on small hydropower and fisheries, may 1-3, 251-256.

Rizzo B., 1968. Fish passage facilities design parameters for Connecticut river dams. Holyoke dam. Bureau of Sport Fisheries and Wildlife, Boston, Massachussets, $40 \mathrm{p}$.

Rizzo B., 1969. Fish passage facilities design parameters for Connecticut river dams. Turners Falls dam. Bureau of Sport Fisheries and Wildlife, Boston, Massachussets, $33 \mathrm{p}$.

Rizzo B., 1986. Fish passage ways and diversion facilities course. Hilton Inn, Merrimack, New Hampshire, 4-8 août 1986, 25 p.

Travade F., 1982. Ascenseur à poissons. Critères de conception. Applications au barrage de Golfech. E.D.F. Direction des Etudes et Recherches. Département Environnement Aquatique et Atmosphérique. HE/31-82-48, $36 \mathrm{p}$.

Travade F., 1990. Monitoring techniques for fish passes recently used in France. International Symposium on Fishways'90 in Gifu, Japan, october 810, 119-126. 